\title{
Path-integral quantum cosmology: A class of exactly soluble scalar-field minisuperspace models with exponential potentials
}

\author{
Luis J. Garay, * Jonathan J. Halliwell, and Guillermo A. Mena Marugán* \\ Center for Theoretical Physics, Laboratory of Nuclear Science, Massachusetts Institute of Technology, \\ Cambridge, Massachusetts 02139
}

(Received 6 December 1990)

\begin{abstract}
We study a class of minisuperspace models consisting of a homogeneous isotropic universe with a minimally coupled homogeneous scalar field with a potential $\alpha \cosh (2 \phi)+\beta \sinh (2 \phi)$, where $\alpha$ and $\beta$ are arbitrary parameters. This includes the case of a pure exponential potential $\exp (2 \phi)$, which arises in the dimensional reduction to four dimensions of five-dimensional Kaluza-Klein theory. We study the classical Lorentzian solutions for the model and find that they exhibit exponential or power-law inflation. We show that the Wheeler-DeWitt equation for this model is exactly soluble. Concentrating on the two particular cases of potentials $\cosh (2 \phi)$ and $\exp (2 \phi)$, we consider the Euclidean minisuperspace path integral for a propagation amplitude between fixed scale factors and scalar-field configurations. In the gauge $\dot{N}=0$ (where $N$ is the rescaled lapse function), the path integral reduces, after some essentially trivial functional integrations, to a single nontrivial ordinary integral over $N$. Because the Euclidean action is unbounded from below, $N$ must be integrated along a complex contour for convergence. We find all possible complex contours which lead to solutions of the Wheeler-DeWitt equation or Green's functions of the Wheeler-DeWitt operator, and we give an approximate evaluation of the integral along these contours, using the method of steepest descents. The steepest-descent contours may be dominated by saddle points corresponding to exact solutions to the full Einstein-scalar equations which may be real Euclidean, real Lorentzian, or complex. We elucidate the conditions under which each of these different types of solution arise. For the $\exp (2 \phi)$ potential, we evaluate the path integral exactly. Although we cannot evaluate the path integral in closed form for the $\cosh (2 \phi)$ potential, we show that for particular $N$ contours the amplitude may be written as a given superposition of exact solutions to the Wheeler-DeWitt equation. By choosing certain initial data for the path-integral amplitude we obtain the amplitude specified by the "no-boundary" proposal of Hartle and Hawking. We discuss the nature of the geometries corresponding to the saddle points of the no-boundary amplitude. We identify the set of classical solutions this proposal picks out in the classical limit.
\end{abstract}

\section{INTRODUCTION}

Most studies in quantum cosmology concentrate on the Dirac quantization procedure, in which the wave function of the universe is found by solving the WheelerDeWitt equation and momentum constraints

$$
\mathscr{H} \Psi=0, \quad H_{i} \Psi=0
$$

Here, $\Psi\left[h_{i j}, \phi, \Sigma\right]$ is the wave function for a compact three-surface $\Sigma$ on which the three-metric is $h_{i j}$ and the matter-field configuration is $\phi$ (Refs. 1 and 2). A more general and powerful tool for calculating the wave function is the path integral, in terms of which the wave function may be written

$\Psi\left[h_{i j}, \phi, \Sigma\right]=\int \mathcal{D} g_{\mu \nu} \mathcal{D} \Phi \exp \left(-I_{g}\left[g_{\mu \nu}\right]-I_{m}\left[g_{\mu \nu}, \Phi\right]\right) ，$

where $I_{m}\left[g_{\mu \nu}, \Phi\right]$ is a matter action and $I_{g}\left[g_{\mu \nu}\right]$ is the Euclidean Einstein-Hilbert action for gravity:

$$
\begin{aligned}
I\left[g_{\mu \nu}\right]= & \frac{1}{16 \pi G} \int_{M} d^{4} x g^{1 / 2}(-R+2 \Lambda) \\
& -\frac{1}{8 \pi G} \int_{\partial M} d^{3} x h^{1 / 2} K .
\end{aligned}
$$

The integral is taken over a certain class of metrics $g_{\mu \nu}$ and matter fields $\Phi$, and over some class of manifolds $M$ with boundary $\partial M$, such that $\Sigma$ is part of $\partial M$, and such that $g_{\mu v}$ and $\Phi$ induce $h_{i j}$ and $\phi$ on $\Sigma$. To make an expression such as (1.2) well defined, it is necessary to introduce the usual gauge-fixing machinery, but also, because the action (1.3) is unbounded from below on real fourmetrics, it is necessary to choose a suitable complex contour. $^{3-10}$ Once these things are done, it may be shown that wave functions defined by (1.2) satisfy the constraint equations (1.1)..$^{11-13}$

The path integral (1.2) is generally a technically more difficult tool to work with than the Wheeler-DeWitt equation, and it is perhaps for this reason that it has received less attention than the constraints (1.1). There are, however, at least two reasons why it is of interest to study the path-integral representation of the wave function. First, it is more general than the canonical quantization 
procedure in that it may be used to construct amplitudes more general than wave functions, ${ }^{2}$ and also that it does not necessarily require a notion of time. ${ }^{14}$ Secondly, certain boundary-condition proposals for the wave function of the Universe are given in path-integral form. In particular, the "no-boundary" proposal of Hartle and Hawking ${ }^{15,16}$ is defined by taking the class of manifolds in (1.2) to be compact manifolds $M$ whose only boundary is $\Sigma$, i.e., $\partial M=\Sigma$. Certain forms of the "tunneling" proposal of Linde and of Vilenkin are also given in path-integral form. ${ }^{17,18}$

Because of the considerable difficulties attending the full field-theoretic expressions of the form (1.1) and (1.2), much attention in quantum cosmology has been lavished on so-called minisuperspace models. ${ }^{19}$ These are models in which the metric and matter fields are severely restricted so that they depend only on a finite number of functions of one variable. Typically, one restricts the (Euclidean) four-metric to be of the form

$$
d s^{2}=N^{2}(\tau) d \tau^{2}+h_{i j} d x^{i} d x^{j},
$$

where $N$ is the lapse function, and the three-metric $h_{i j}$ is restricted in such a way that it depends on a finite number of functions of $\tau, q^{\alpha}(\tau)$, say, where $\alpha=1, \ldots, n$. With these restrictions, the action (1.3) will then typically be of the form

$$
I[q(\tau)]=\int_{0}^{1} d \tau N\left(\frac{1}{2 N^{2}} f_{\alpha \beta} \dot{q}^{\alpha} \dot{q}^{\beta}+U(q)\right) .
$$

Here we have used the freedom to shift $\tau$ and rescale $N$ to set the initial and final values of $\tau$ to be 0 and 1, respectively. $f_{\alpha \beta}(q)$ is the metric on minisuperspace and has indefinite signature [one minus sign and $(n-1)$ plus signs]. An action of the form (1.5) will also be obtained if, as is often convenient, the lapse function is rescaled by some function of the three-metric $h_{i j}$. Equation (1.5) has the form of the action for a relativistic point article with spacetime-dependent mass squared $U(q)$ moving in a curved $n$-dimensional spacetime with metric $f_{\alpha \beta}$.

In Ref. 11, it was shown that the path-integral construction for the propagation amplitude between fixed initial and final $q^{\alpha}$ for systems described by an action of the form (1.5) is, in the gauge $\dot{N}=0$,

$$
G\left(q^{\alpha^{\prime \prime}} \mid q^{\alpha^{\prime}}\right)=\int d N\left\langle q^{\alpha^{\prime \prime}}, N \mid q^{\alpha^{\prime}}, 0\right\rangle .
$$

The integrand of (1.6) is an ordinary quantummechanical propagator between fixed $q^{\alpha}$ in fixed time $N$ :

$$
\left\langle q^{\alpha^{\prime \prime}}, N \mid q^{\alpha^{\prime}}, 0\right\rangle=\int D q^{\alpha} \exp \{-I[q(\tau)]\} .
$$

Because the action $I$ is not bounded from below, it is necessary to integrate $q^{\alpha}(\tau)$ and $N$ along complex contours. The range of the $N$ contour turns out to be of significance. If the $N$ contour is taken to be of infinite length, or closed, then, using the fact that (1.7) satisfies the (Euclidean) Schrödinger equation, one may show that (1.6) is a solution to the Wheeler-DeWitt equation.

$$
\hat{H}^{\prime \prime} G\left(q^{\alpha^{\prime \prime}} \mid q^{\alpha^{\prime}}\right)=\left[-\frac{1}{2} \nabla^{2}+\xi \mathbb{R}+U(q)\right] G\left(q^{\alpha^{\prime \prime}} \mid q^{\alpha^{\prime}}\right)=0 .
$$

Here, $\nabla^{2}$ and $\mathbb{R}$ are the Laplacian and curvature scalar in the metric $f_{\alpha \beta}$ and the coefficient $\xi$ is chosen to be the conformal coupling. ${ }^{11,20,21}$ These choices of operator ordering guarantee invariance under field redefinitions of the $q^{\alpha}$ and rescalings of the lapse function $N$. If the $N$ contour is taken to be of half-infinite length, with one of the endpoints $N=0$, then (1.7) is a Green's function of the Wheeler-DeWitt operator; i.e., one obtains a $\delta$ function on the right-hand side of (1.8).

Given the explicit path integral for minisuperspace, Eq. (1.6), it becomes desirable, as in ordinary quantum mechanics, to build up a repertoire of models for which the path integral can be evaluated exactly, or at least almost exactly. Because of the form of (1.6), this clearly becomes an issue of finding those minisuperspace models which, with a suitable choice of variables, have an action belonging to the well-known class of models for which the ordinary quantum-mechanical propagator (1.7) may be evaluated exactly. For then the propagation amplitude (1.6) is obtained by a single ordinary integral over $N$, which, with a bit of luck, may be evaluated exactly, or, if this is not possible, evaluated approximately using the method of steepest descents.

A number of such models have recently been found. Reference 6 considered the de Sitter minisuperspace model, a one-dimensional homogeneous isotropic model described by a single scale factor. Some anisotropic models with two scale factors were studied in Ref. 8. A homogeneous isotropic model with a massless scalar field, in $2+1$ dimensions, was considered in Ref. 10. This model is of interest in relation to wormholes. Models with conformal scalar fields are also exactly soluble. ${ }^{22}$ Some Bianchi type-I models, but with a different type of gauge fixing than in (1.6), were considered in Ref. 23.

The purpose of the present paper is to study a class of homogeneous isotropic minisuperspace models with a minimally coupled scalar field matter source in which the potential is such that the path integral may be solved exactly, or almost exactly. The minisuperspace will, therefore, be two dimensional, described by a scale factor $a$ and scalar field $\phi$. Unfortunately, the most interesting potentials from the cosmological point of view, such as chaotic inflation potentials or the Coleman-Weinberg potential, do not generally lead to models that can be solved exactly, even at the classical level. Here, however, we will study the class of models for which the scalar field potential is

$$
V(\phi)=\alpha \cosh (2 \phi)+\beta \sinh (2 \phi),
$$

where $\alpha$ and $\beta$ are arbitrary parameters, and the (dimensionless) field $\phi$ is related to the standard scalar field $\Phi$ by $\phi=(4 \pi G / 3)^{1 / 2} \Phi$. We will show that the minisuperspace path integral can be evaluated exactly, or almost exactly (depending on the values of $\alpha$ and $\beta$, and on the precise amplitude one is interested in).

The class of potentials (1.9) is not entirely without physical content. For large $|\phi|, V(\phi) \sim \exp ( \pm 2 \phi)$. Pure exponential potentials of this type are known to lead to power-law inflation. ${ }^{24-26}$ Furthermore, a potential of exactly the form $\exp (2 \phi)$ arises in the dimensional reduction to four dimensions of five-dimensional pure gravity 
Kaluza-Klein theory ${ }^{24,27}$ with cosmological constant. A1though the interest of this paper is somewhat mathematical, we shall restrict to ranges of $\alpha$ and $\beta$ in (1.9) that are reasonably physical. In particular, we restrict $\alpha$ and $\beta$ to be such that $\alpha>0$ and $\alpha \geq|\beta|$, so that the potential is bounded from below. Although we can solve the model for any $\alpha$ and $\beta$, for convenience we will consider only the two cases $\alpha=\beta$, for which $V(\phi)=\alpha \exp (2 \phi)$, and $\beta=0$, for which $V(\phi)=\alpha \cosh (2 \phi)$. All other cases satisfying the above restrictions are qualitatively the same as one of these two special cases and can, in fact, be transformed into these cases by simple field redefinitions. There are unfortunately no values of $\alpha$ and $\beta$ for which $V(\phi)$ is both bounded from below and has its minimum at zero.

The case of the $\cosh (2 \phi)$ potential is closely related to the de Sitter minisuperspace model studied in Ref. 6, which consisted of a homogeneous isotropic model with no scalar field but with a cosmological constant. In fact, it reduces to it precisely if $\phi$ is set to zero. We will find in what follows that the analysis is similar, but of perhaps greater interest for the following reason. The de Sitter model could be criticized on the grounds of not possessing any true dynamics in that, because the theory is a parametrized theory and the single variable describing it, the scale factor, is not really dynamical but is "time." This model improves the situation in that the inclusion of a scalar field leads to nontrivial dynamical content.

We begin in Sec. II by describing the model. We write down the Wheeler-DeWitt equation and show that exact solutions exist. In Sec. III we describe the properties of the classical Lorentzian solutions. In Sec. IV we consider the path integral between fixed $a$ and $\phi$. It readily reduces to a single ordinary integral over the lapse function $N$. We are not, in fact, able to evaluate the integral exactly for general values of $\alpha$ and $\beta$, so we perform a steepest-descent analysis. We begin by finding the saddle points of the $N$ integral. These correspond to exact solutions to the full Einstein-scalar field equations, satisfying the boundary conditions implied by the path integral. These exact solutions may be real Euclidean, real Lorentzian, or complex, depending on the relative location in minisuperspace of the initial and final points of the boundary-value problem. We construct a series of pictures showing the conditions under which each of these different types of solutions arise.

In Sec. V, we find the steepest-descent contours passing through the saddle points, and evaluate the integral approximately along various contours of interest. We also show that the integral may be written as a weighted integral over Airy functions. We evaluate the amplitude exactly for the case of the $\exp (2 \phi)$ potential.

In Sec. VI we attempt to implement the no-boundary proposal of Hartle and Hawking. We discuss the initial conditions implied for the propagation amplitude. We find the no-boundary wave function to at least leading order in the saddle-point approximation. We discuss the nature of the geometries and matter-field configurations corresponding to the saddle points in the no-boundary amplitude. We identify the regions of configuration space in which the no-boundary wave function is oscilla- tory, thereby predicting classical spacetime, and we discuss the nature of the classical Lorentzian solutions picked out by this proposal. We summarize and conclude in Sec. VII.

\section{THE SCALAR-FIELD MODELS}

We will take the gravitational part of the action for the model to be the Einstein-Hilbert action with a boundary term (1.3). We restrict the Euclidean metric to be homogeneous and isotropic, of the form

$$
d s^{2}=\sigma^{2}\left(N^{2} \frac{d \tau^{2}}{a^{2}(\tau)}+a^{2}(\tau) d \Omega_{3}^{2}\right),
$$

where $\sigma^{2}=2 G / 3 \pi$ and $d \Omega_{3}^{2}$ is the metric on the unit three-sphere. The unconventional lapse rescaling in (2.1) simplifies the algebra. As mentioned in the previous section, the quantization procedure is insensitive to such rescalings if the operator ordering in the Wheeler-DeWitt equation is appropriately chosen. ${ }^{11,20,21}$ For the matter source we take a minimally coupled scalar field $\Phi$ with potential $\widetilde{V}(\Phi)$, with Euclidean action

$$
I_{m}=\frac{1}{2} \int d^{4} x g^{1 / 2}\left[g^{\mu \nu} \partial_{\mu} \Phi \partial_{v} \Phi+\widetilde{V}(\Phi)\right] \text {. }
$$

We restrict $\Phi$ to be homogeneous. Writing

$$
\Phi=\left(2 \pi^{2} \sigma^{2}\right)^{-1 / 2} \phi(t)
$$

and

$$
\widetilde{V}(\Phi)=\left(2 \pi^{2} \sigma^{4}\right)^{-1} V(\phi),
$$

the total Euclidean action for the model in terms of $a$ and $\phi$ is

$$
I=\frac{1}{2} \int_{0}^{1} d \tau N\left[-\frac{a^{2} \dot{a}^{2}}{N^{2}}+\frac{a^{4} \dot{\phi}^{2}}{N^{2}}+a^{2} V(\phi)-1\right]
$$

Even for the simplest choices of potential $V(\phi)$, this system is generally very difficult, if not impossible, to solve exactly, even at the classical level. The following change of variables turns out to be very useful. Let

$$
x=a^{2} \cosh (2 \phi), \quad y=a^{2} \sinh (2 \phi) .
$$

The action (2.3) then simplifies to

$I=\frac{1}{2} \int_{0}^{1} d \tau N\left[\frac{1}{4 N^{2}}\left(-\dot{x}^{2}+\dot{y}^{2}\right)+f(x, y)-1\right]$,

where $f(x, y)=a^{2} V(\phi)$. The object now is to choose $V(\phi)$ in such a way that $f(x, y)$ takes a form sufficiently simple to permit a detailed analysis of the system described by (2.5). Freedom to choose $V(\phi)$ does not give complete freedom to choose $f(x, y)$, however. It is not difficult to see that $f$ must satisfy

$$
f(c x, c y)=c f(x, y)
$$

for any $c$. This means that $f$ must be of the form $f(x, y)=x g(x / y)$, where $g$ is an arbitrary function. One of the simplest examples of a function of this form is $f=a x+\beta y$, for which the system described by (2.5) is indeed very simple. The corresponding scalar field poten- 
tial is

$$
V(\phi)=\alpha \cosh (2 \phi)+\beta \sinh (2 \phi) .
$$

As stated in the Introduction, this is the class of potentials we are going to study in this paper. We are therefore going to study the system described by the action

$I=\frac{1}{2} \int_{0}^{1} d \tau N\left[\frac{1}{4 N^{2}}\left(-\dot{x}^{2}+\dot{y}^{2}\right)+\alpha x+\beta y-1\right]$.

We will concentrate on the cases $\alpha=\beta$ and $\beta=0$, as explained in the Introduction. In fact, the cases $\alpha>|\beta|$ are related to the case of the $\cosh (2 \phi)$ potential by a simple transformation. Under the "Lorentz" transformation

$$
\widetilde{x}=\frac{\alpha x+\beta y}{\left(a^{2}-\beta^{2}\right)^{1 / 2}}, \quad \tilde{y}=\frac{\alpha y+\beta x}{\left(a^{2}-\beta^{2}\right)^{1 / 2}},
$$

the action (2.7) is turned into one of identical form, but with $x, y$ replaced by $\tilde{x}, \tilde{y}, \alpha$ replaced by $\left(\alpha^{2}-\beta^{2}\right)^{1 / 2}$, and $\beta=0$.

It is conceivable that there are other scalar-field potentials that could be handled using the change of variables (2.4), and this is a possibility worth pursuing. We also note that an action very similar to (2.7) may be obtained in approximations to more general minisuperspace models with two variables. Using the fact that all twodimensional minisuperspaces are conformally flat, by a suitable lapse rescaling one can always cast the minisuperspace action in the form (2.5), but with $f(x, y)-1$ replaced by some potential $U(x, y)$. An action very similar to $(2.7)$ is then obtained by linearizing $U(x, y)$ about a particular point, and this could be used in studying the minisuperspace version of the thin-sandwich problem. Similar remarks apply to $n$-dimensional minisuperspace models that are conformally flat.

Some remarks are in order concerning the domain of the variables $x, y$ defined by (2.4). The physically interesting domain of $a$ and $\phi$ is the region $a \geq 0,-\infty<\phi<\infty$. Under the transformation (2.4), this region is mapped into the "future lightcone" of the origin in $x y$ space (plotting the timelike variable $x$ vertically), i.e., the region $x<|y|$. The following question then naturally arises: Should one insist in the quantum theory that everything takes place in this domain? At the level of the WheelerDeWitt equation, the issue of quantizing with variables on a restricted range is a subtle one that we do not address. ${ }^{28}$ Our main concern in this paper, however, is the semiclassical evaluation of the path integral. As has been realized elsewher $\mathrm{e}^{5-10}$ and as will be seen here, in studying the saddlepoints of a path integral of the form (1.2) or (1.6), one is inevitably led to complex solutions to the field equations (although one normally takes the endpoints to be real, because these correspond to the argument of the wave function). For our purposes, it is therefore most appropriate to think of $a$ and $\phi$ as being completely unrestricted complex numbers. Any other coordinates that are analytic functions of $a$ and $\phi$, such as $x$ and $y$, will be equally good. In what follows, we will therefore impose no restrictions on $x$ and $y$. This will have some consequences, as we shall see later.

Varying the action (2.7) with respect to $x, y$, and $N$, one obtains the second-order (Euclidean) field equations and constraint

$$
\begin{aligned}
& \ddot{x}=-2 \alpha N^{2}, \ddot{y}=2 \beta N^{2}, \\
& \frac{1}{4 N^{2}}\left(\dot{x}^{2}-\dot{y}^{2}\right)+\alpha x+\beta y-1=0 .
\end{aligned}
$$

It is straightforward to show that the Wheeler-DeWitt equation for the model is

$$
\left(4 \frac{\partial^{2}}{\partial x^{2}}-4 \frac{\partial^{2}}{\partial y^{2}}+\alpha x+\beta y-1\right) \Psi(x, y)=0 .
$$

It is readily solved by separation of variables. The solutions are of the form

$$
\Psi(x, y)=[b \operatorname{Ai}(X)+c \operatorname{Bi}(X)][d \operatorname{Ai}(Y)+e \operatorname{Bi}(Y)],
$$

where $\mathrm{Ai}$ and $\mathrm{Bi}$ are Airy functions, ${ }^{29} b, c, d$ and $e$ are arbitrary constants, and

$$
\begin{aligned}
& X=(1+\mu-\alpha x)(2 \alpha)^{-2 / 3}, \\
& Y=(\beta y+\mu)(2 \beta)^{-2 / 3},
\end{aligned}
$$

$\mu$ is the separation constant. For $X<<1, Y>>1$, for example, the asymptotic form of (2.12) is a sum of terms of the form

$$
\begin{aligned}
\Psi(x, y) \sim & \exp \left[ \pm \frac{i}{3 \alpha}\left(\alpha a^{2} \cosh 2 \phi-\mu-1\right)^{3 / 2}\right] \\
& \times \exp \left[ \pm \frac{1}{3 \beta}\left(\beta a^{2} \sinh 2 \phi+\mu\right)^{3 / 2}\right] .
\end{aligned}
$$

If $\beta=0$ [i.e., $V(\phi)=\alpha \cosh 2 \phi$ ], the solutions are of the form

$$
\Psi(x, y)=[b \mathrm{Ai}(X)+c \operatorname{Bi}(X)] \exp \left[ \pm \frac{\mu^{1 / 2}}{2} y\right),
$$

and, for $X<1$,

$$
\begin{aligned}
\Psi(x, y) \sim & \exp \left[ \pm \frac{i}{3 \alpha}\left(\alpha a^{2} \cosh 2 \phi-\mu-1\right)^{3 / 2}\right] \\
& \times \exp \left[ \pm \frac{\mu^{1 / 2}}{2} a^{2} \sinh 2 \phi\right] .
\end{aligned}
$$

Although it is possible to find exact solutions to the Wheeler-DeWitt equation, it turns out that the particular object we are interested in calculating using the path integral - the propagation amplitude between fixed $x$ and $y$-cannot, in fact, be evaluated exactly for the case of the $\cosh (2 \phi)$ potential, as we shall see in the following sections. It may, however, be expressed as an infinite sum over $\mu$ of the above exact solutions.

To end this section, it is appropriate to make some remarks about minisuperspace. There is no known sense in which minisuperspace is the first step in a systematic approximation to a full theory of quantum gravity, should it exist. Indeed, there are indications that it may give quite misleading results if regarded as such. ${ }^{30}$ However, stud- 
ies of minisuperspace models may be argued to be partially relevant to a full quantum theory of gravity. The minisuperspace action (2.7) was obtained by inserting Ansätze for $g_{\mu \nu}$ and $\Phi$ into the Einstein-scalar action. The extrema of the action (2.7) are the solutions to the secondorder field equations and constraint (2.9) and (2.10). One may verify that these field equations coincide with those obtained by inserting the metric and matter Ansätze directly into the full Einstein-scalar field equations (although this is not guaranteed - the acts of varying and constraining generally do not commute). All solutions to the field equations (2.9) and (2.10) are therefore solutions to the full Einstein-scalar field equations and, in particular, the minisuperspace action of such solutions will coincide with the full Einstein action of these solutions. What this means is that the lowest-order saddle-point approximation to the minisuperspace path integral, in which one writes the amplitude as $\exp \left(-I_{\mathrm{cl}}\right)$, where $I_{\mathrm{cl}}$ is the action of the saddle-point, will coincide with the lowest-order saddle-point approximation to the full path integral (1.2). It is in this sense that minisuperspace models are partially relevant to a full quantum theory of gravity.

\section{CLASSICAL LORENTZIAN SOLUTIONS}

We now discuss the classical Lorentzian solutions of the system under consideration. Introducing $v$ defined by $a=e^{v}$ and working in the gauge $N=e^{-\phi}$, we write the Lorentzian metric in the form

$$
d s^{2}=\sigma^{2}\left(-e^{-2 \phi} d t^{2}+e^{2 v} d \Omega_{3}^{2}\right)
$$

We also define

$$
w \equiv \dot{v}=\frac{\dot{a}}{a,} \quad z \equiv \dot{\phi} .
$$

The second-order Lorentzian field equations may then be written

$$
\begin{aligned}
& \dot{w}=-w^{2}-z w-2 z^{2}+\frac{\alpha+\beta}{2}+\frac{\alpha-\beta}{2} e^{-4 \phi}, \\
& \dot{z}=-3 w z-z^{2}-\frac{\alpha+\beta}{2}+\frac{\alpha-\beta}{2} e^{-4 \phi},
\end{aligned}
$$

and the constraint is

$$
w^{2}-z^{2}-\frac{\alpha+\beta}{2}-\frac{\alpha-\beta}{2} e^{-4 \phi}+k e^{-2 \phi-2 v}=0 .
$$

Here, we have introduced the curvature $k$ of the threemetric $d \Omega_{3}^{2}$. We are interested mainly in the three-sphere case, $k=+1$, but for completeness we briefly retain the cases $k=0,-1$ also. As stated in the previous section, we restrict our attention to the cases of a pure exponential or a $\cosh (2 \phi)$ potential.

Consider first the pure exponential case, $\alpha=\beta$. In this case the second-order field equations (3.3) are then a first-order plane autonomous system. The classical solutions to this system have previously been studied in Refs. 24 and 25 in relation to power-law inflation. The phase portrait of the system is shown in Fig. 1. The constraint equation (3.4) is used in the construction of the phase

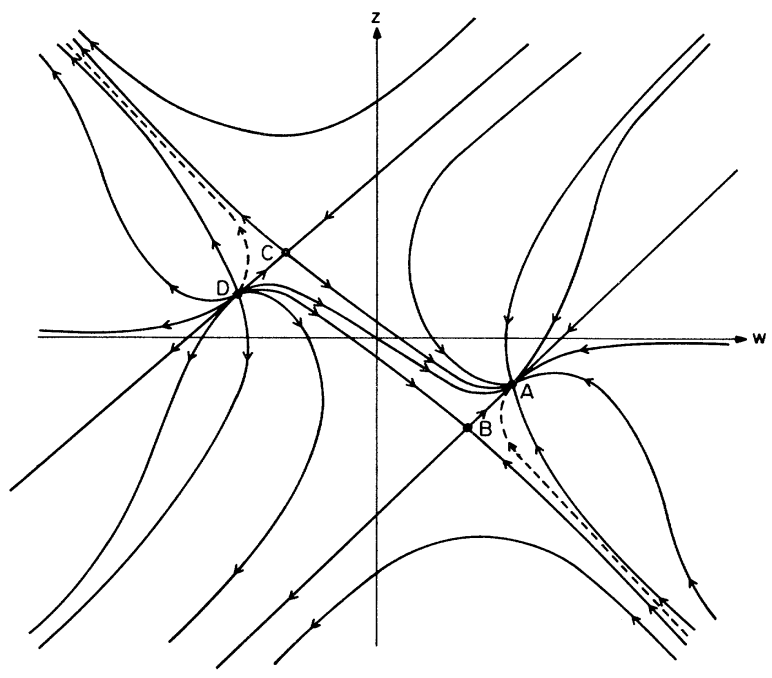

FIG. 1. The two-dimensional phase portrait for the exponential potential. Here $z=\dot{\phi}$ and $w=\dot{a} / a$. The $k=+1$ solutions we are interested in here lie between the two branches of the hyperbola $w^{2}-z^{2}=\alpha$.

portrait only to establish that the relevant region is the region $w^{2}-z^{2} \leq \alpha$ (solutions lying outside this region correspond to the $k=-1$ solutions, and the $k=0$ solutions lie on the boundary of this region). There is only one stable critical point (point $A$ in Fig. 1), with coordinates

$$
(w, z)=\left(\frac{3 \sqrt{2 \alpha}}{4},-\frac{\sqrt{2 \alpha}}{4}\right) .
$$

There are three other critical points, labeled $B, C$, and $D$, which are unstable and have coordinates, respectively,

$$
\begin{aligned}
& {\left[\frac{\sqrt{2 \alpha}}{2},-\frac{\sqrt{2 \alpha}}{2}\right),} \\
& {\left[-\frac{\sqrt{2 \alpha}}{2},+\frac{\sqrt{2 \alpha}}{2}\right),} \\
& {\left[-\frac{3 \sqrt{2 \alpha}}{4},+\frac{\sqrt{2 \alpha}}{4}\right) .}
\end{aligned}
$$

The main feature of the phase portrait is that a certain proportion of the solutions are attracted to the critical point $A$, Eq. (3.5). Introducing Robertson-Walker time $\widetilde{t}=\int d t e^{-\phi}$, it is readily shown that, at point $A$, one has $a(\widetilde{t}) \sim \widetilde{t}^{3}$. This is an example of power-law inflation. ${ }^{25}$

Now consider the $\cosh (2 \phi)$ potential case, $\beta=0$. The second-order field equations (3.3) together with the relation $\dot{\phi}=z$ form a three-dimensional first-order autonomous system. It has only two critical points. There is one stable node at

$$
\phi=0, \quad z=0, \quad w=\sqrt{\alpha}
$$

and one unstable node at 


$$
\phi=0, \quad z=0, \quad w=-\sqrt{\alpha} .
$$

The solutions to the linearized system around the stable node (3.7) are of the general form

$$
\begin{aligned}
& \phi=b e^{-\sqrt{\alpha} t}+c e^{-2 \sqrt{\alpha}}, \\
& w=\sqrt{\alpha}-\sqrt{\alpha} b e^{-\sqrt{\alpha} t}+d e^{-2 \sqrt{\alpha} t},
\end{aligned}
$$

where $b, c$, and $d$ are arbitrary constants. For any initial value of $v$, the stable solution (3.7) satisfies the constraint (3.4) asymptotically. Therefore, (3.7) is an asymptotically stable solution for the whole system of dynamical equations, plus the constraint equation. It is easily seen that the solution (3.7) is the standard exponential inflation $a \sim e^{\alpha \tilde{t}}$.

Because it is three dimensional, the phase portrait of the system in $(x, z, \phi)$ space is rather difficult to construct. Nevertheless, one can get some idea of the structure of this three-dimensional portrait by studying constant $\phi$ slices. It is readily shown that each constant $\phi$ slice is, in fact, qualitatively the same as the phase portrait for the exponential potential, differing only in the exact location of the critical points. It is, however, important to understand the significance of the trajectories in each constant $\phi$ slice. They do not in any sense correspond to trajectories of the full three-dimensional system; rather, they represent the instantaneous direction of the trajectories of the three-dimensional system at a fixed value of $\phi$.

The section $\phi=+\infty$ is obtained by replacing $\alpha$ by $\alpha / 2$ in Fig. 1. As $\phi$ decreases from $+\infty$, the corresponding critical points $A$ and $B$ move apart from each other along the straight line $z=w-\sqrt{\alpha}$, while the critical points $C$ and $D$ separate along the parallel line $z=w+\sqrt{\alpha}$. Note that, for all of these critical points, the vector $(\dot{w}, \dot{z})$ vanishes, but $\dot{\phi}$ does not, in general, so that these critical points do not correspond to critical points of the whole three-dimensional portrait - only of the two-dimensional constant $\phi$ slice.

\section{THE PATH INTEGRAL AND COMPLEX SOLUTIONS}

We now consider the path integral for the model. As outlined in Sec. I, the propagation amplitude between fixed $x$ and $y$ has the form

$$
\begin{aligned}
& G\left(x^{\prime \prime}, y^{\prime \prime} \mid x^{\prime}, y^{\prime}\right) \\
& \quad=\int d N \int \mathcal{D} x \mathscr{D} y \exp \{-I[x(\tau), y(\tau), N]\},
\end{aligned}
$$

where $I$ is the action (2.7). ${ }^{11}$ The measure $\mathcal{D} x \mathscr{D} y$ is the standard quantum-mechanical one for flat configuration spaces such as this. The sum is taken over the class of histories $(x(\tau), y(\tau), N)$ satisfying the boundary conditions

$x(0)=x^{\prime}, \quad y(0)=y^{\prime}, \quad x(1)=x^{\prime \prime}, \quad y(1)=y^{\prime \prime}$.

Following Refs. 6 and 8, the integral may be evaluated using the following procedure. Let

$$
x(\tau)=\bar{x}(\tau)+X(\tau), \quad y(\tau)+Y(\tau),
$$

where $(\bar{x}(\tau), \bar{y}(\tau))$ are the solutions to the second-order field equations (2.9), but they do not satisfy the constraint
(2.10). Explicitly, they are given by

$$
\begin{aligned}
& \bar{x}(\tau)=-\alpha N^{2} \tau^{2}+\left(x^{\prime \prime}-x^{\prime}+\alpha N^{2}\right) \tau+x^{\prime}, \\
& \bar{y}(\tau)=\beta N^{2} \tau^{2}+\left(y^{\prime \prime}-y^{\prime}-\beta N^{2}\right) \tau+y^{\prime} .
\end{aligned}
$$

In terms of the variables (4.3), the action may be written

$$
\begin{aligned}
I[x(\tau), y(\tau), N]= & I_{0}\left(x^{\prime \prime}, y^{\prime \prime}, N \mid x^{\prime}, y^{\prime}, 0\right) \\
& +I_{2}[X(\tau), Y(\tau), N] .
\end{aligned}
$$

Here, $I_{0}$ is the action of the solution to the second-order field equations (4.4) and (4.5) and is given by

$$
\begin{aligned}
I_{0}= & \frac{\alpha^{2}-\beta^{2}}{24} N^{3}+\frac{1}{4}\left[\alpha\left(x^{\prime \prime}+x^{\prime}\right)+\beta\left(y^{\prime \prime}+y^{\prime}\right)-2\right] N \\
& +\frac{-\left(x^{\prime \prime}-x^{\prime}\right)^{2}+\left(y^{\prime \prime}-y^{\prime}\right)^{2}}{8 N} .
\end{aligned}
$$

$I_{2}$ is given by

$$
I_{2}[X(\tau), Y(\tau)]=\frac{1}{8 N} \int_{0}^{1} d \tau\left(-\dot{X}^{2}+\dot{Y}^{2}\right) .
$$

Equation (4.1) may now be written

$$
\begin{aligned}
& G\left(x^{\prime \prime}, y^{\prime \prime} \mid x^{\prime}, y^{\prime}\right) \\
& \quad=\int d N \exp \left(-I_{0}\right) \int \mathscr{D} X \mathscr{D} Y \exp \left(-I_{2}\right) .
\end{aligned}
$$

For each complex value of $N$ in $I_{2}$, it is straightforward to choose convergent complex contours for the integrals over $X$ and $Y$, and one obtains the result

$$
\begin{aligned}
& G\left(x^{\prime \prime}, y^{\prime \prime} \mid x^{\prime}, y^{\prime}\right) \\
& \quad=\int \frac{d N}{N} \exp \left[-I_{0}\left(x^{\prime \prime}, y^{\prime \prime}, N \mid x^{\prime}, y^{\prime}, 0\right)\right] .
\end{aligned}
$$

It is readily verified that (4.10) is an exact solution to the Wheeler-DeWitt equation (2.11) if $N$ is integrated along any convergent contour of infinite length or if it is integrated along a closed contour. Integrating $N$ along a convergent half-infinite contour may be shown to lead to a Green's function of the Wheeler-DeWitt operator; i.e., one obtains $-i \delta\left(x^{\prime \prime}-x^{\prime}\right) \delta\left(y^{\prime \prime}-y^{\prime}\right)$ on the right-hand side of (2.11). The latter fixes overall numerical factors in (4.10), but we will ignore these in what follows. Equation (4.10) is the expression we will be concentrating on in the following analysis.

Let us begin by studying the saddle points of (4.10), corresponding to solutions of the Einstein-scalar field equations. It is convenient to define the quantities

$$
\begin{aligned}
& s^{2}=-\left(x^{\prime \prime}-x^{\prime}\right)^{2}+\left(y^{\prime \prime}-y^{\prime}\right)^{2} \\
& R=\alpha\left(x^{\prime \prime}+x^{\prime}\right)+\beta\left(y^{\prime \prime}+y^{\prime}\right)-2 .
\end{aligned}
$$

Up to constant factors, $s^{2}$ is the geodesic distance in minisuperspace between the initial and final points, and $R$ is the parameter time average of the potential $U=\alpha x+\beta y-1$ along that geodesic. $s^{2}$ will therefore be positive or negative if the initial and final points are, respectively, spacelike or timelike separated. $R$ will be positive or negative depending on whether the connecting geodesic lies "largely" in the region of, respectively, a 
positive or negative potential. In terms of $s^{2}$ and $R$, Eq. (4.7) may be written

$$
I_{0}=\frac{a^{2}-\beta^{2}}{24} N^{3}+\frac{R}{4} N+\frac{s^{2}}{8 N} .
$$

The saddle points are the values of $N$ for which $\partial I_{0} /(\partial N)=0$.

Consider first the case of the purely exponential potential for which $\alpha=\beta$. Then the coefficient of $N^{3}$ vanishes in (4.12), and there are two saddle points at the values of $N$ given by

$$
N= \pm\left(\frac{s^{2}}{2 R}\right)^{1 / 2}
$$

Inserting these values of $N$ into the solutions (4.4) and (4.5), one thus obtains exact solutions to the Einsteinscalar field equations satisfying the boundary conditions (4.2). These solutions are real Euclidean if $s^{2}>0$ and $R>0$, or if $s^{2}<0$ and $R<0$. Similarly, they are real Lorentzian if $s^{2}<0$ and $R>0$, or if $s^{2}>0$ and $R<0$.

As a brief aside, we should remark that we are allowing ourselves a little license in our usage of the words "Euclidean" and "Lorentzian" in the above context. Here, and in what follows, we take these words to mean that the lapse [and therefore the action (4.12)] is real or imaginary, respectively. The possible difficulty with this, however, is that it does not necessarily follow that the corresponding metric (2.1) in terms of $a$ is Euclidean or Lorentzian. The point is that, although the solution in terms of $x$ and $y,(4.4)$ and (4.5), will be real if $N$ is real or imaginary, the inverse of the transformation (2.4) yields $a^{2}=\left(x^{2}-y^{2}\right)^{1 / 2}$, and $a$ will not be real if the solution goes outside the light cone in $x y$ space, as indeed it may. We will discuss this point further in Sec. VI.

Now consider the case of the $\cosh (2 \phi)$ potential for which $\beta=0$. There are four saddle points at the points for which

$$
\alpha^{2} N^{2}=-R \pm\left(R^{2}+\alpha^{2} s^{2}\right)^{1 / 2} .
$$

Again, when inserted into (4.4) and (4.5), these lead to exact solutions to the Einstein-scalar boundary-value problem. These solutions may be real Euclidean, real Lorentzian, as above, but in this case they may also be complex. It is useful to note that the discriminant may be written

$R^{2}+\alpha^{2} s^{2}=\alpha^{2}\left(y^{\prime \prime}-y^{\prime}\right)^{2}+4\left(1-\alpha x^{\prime}\right)\left(1-\alpha x^{\prime \prime}\right)$.

This shows that the solution for $N$ can be complex (rather than just pure imaginary) only if the end points lie on opposite sides of the curve $U=0$. One can again explicitly write down the conditions under which these different types of solution arise, but it is clearer to exhibit the results pictorially.

Our object is to produce a set of pictures showing, in the $x y$ plane, the regions corresponding to the sets of final points $\left(x^{\prime \prime}, y^{\prime \prime}\right)$, which are reached by real Euclidean, real Lorentzian, or complex solutions, starting from a given initial point $\left(x^{\prime}, y^{\prime}\right)$. In general, one could, in principle, have a different picture for each initial point. However, the nature of the solutions depends only on the signs of the three quantities $R, s^{2}$, and $\left(R^{2}+\alpha^{2} s^{2}\right) . y^{\prime}$ enters only through $s^{2}$ in the combination $\left(y^{\prime \prime}-y^{\prime}\right)^{2}$. We may therefore restrict to the case $y^{\prime}=0$. The picture for arbitrary $y^{\prime}$ is obtained merely by translating in the $y$ direction. Similarly (although not immediately obvious), the value of $x^{\prime}$ turns out to be significant only inasmuch as it effects the sign of $\left(1-\alpha x^{\prime}\right)$ in the discriminant (4.15). There are therefore only three qualitatively different cases, namely $\alpha x^{\prime}>1, \alpha x^{\prime}=1$, and $\alpha x^{\prime}<1$. So, for example, the picture for any $x^{\prime}$ for which $\alpha x^{\prime}>1$ may be turned into the picture for any other $x^{\prime}$ with $\alpha x^{\prime}>1$ by translation and rescaling in the $x$ direction.

The three qualitatively distinct cases are shown in Figs. 2(a)-2(c). Each figure depicts the type of solutions arising when the initial point is the point at the center of the figure. The $x y$ plane is divided up into regions by the null lines $s^{2}=0$ and the parabola $R^{2}+\alpha^{2} s^{2}=0$. The parabola touches the null lines at the points at which $R=0$. There are four cases, denoted I, II, III, and IV in the figures.

Case I: $s^{2}>0$. There are two real solutions and two imaginary solutions.

Case II: $R^{2}+\alpha^{2} s^{2}<0$. All four solutions are complex. There are no complex solutions in the case $\alpha x^{\prime}=1$, Fig. 2(c).

Case III: $s^{2}<0, R^{2}+\alpha^{2} s^{2}>0, R<0$. All four solutions are real.

Case IV: $s^{2}<0, R^{2}+\alpha^{2} s^{2}>0, R>0$. All four solutions are imaginary.

From these figures, one may see at a glance what type of solutions arise for given initial and final data. One particular feature of the solutions is quite striking: when the initial and final points are sufficiently close together in minisuperspace, they can always be connected by a real Euclidean or real Lorentzian solution (in the sense explained above). It is only when the points are more than a certain finite distance apart that the only solutions are genuinely complex ones. A reasonable conjecture (that we do not attempt to justify) is that the same is also true in the full Einstein equations.

Finally, we make the following remarks about complex solutions. When a complex solution arises in a problem such as this, it is sometimes possible to think of the solution as a combination of a real Euclidean solution and a real Lorentzian solution (where here, real Euclidean and real Lorentzian have their strict meaning, i.e., that the four-metric is real Euclidean or real Lorentzian, with real matter fields). A solution of this type is sometimes known as a "real tunneling geometry," and some examples may be found in Ref. 31. Complex solutions may often be simplified by performing a complex diffeomorphism on $\tau$ which preserves the end points, $\tau=0$ and 1 (Ref. 5). Such a diffeomorphism will preserve the value of the action of the solution and thus, for the purposes of semiclassical approximation to path integral, the transformed solution may be regarded as equivalent. For a complex solution to be a real tunneling geometry, it is necessary that there exist a complex diffeomorphism of the solution $\tau \rightarrow z(\tau)$ say, such that, in the transformed solution, the geometry is real Euclidean for $0<\tau<\tau$ for some $\widetilde{\tau}$, and real Lorentzian for $\widetilde{\tau}<\tau<1$, and further- 
more, it is necessary that both the extrinsic curvature $K_{i j}$ and the normal derivatives of any matter fields all vanish on the transitional surface labeled by $\widetilde{\tau}$ (so that the solution has a finite action).

With these comments in mind, consider the complex solutions obtained here for the $\cosh (2 \phi)$ model. The solu- tion for $y(\tau)$ has $\dot{y}$ equal to a constant. $\dot{y}$ can therefore never vanish, nor can any transformed $y$ (under a wellbehaved diffeomorphism of $\tau$ ). This means that the complex solutions we have found are genuine complex solutions to the Einstein-scalar boundary-value problem and are not in any sense equivalent to real tunneling solu-

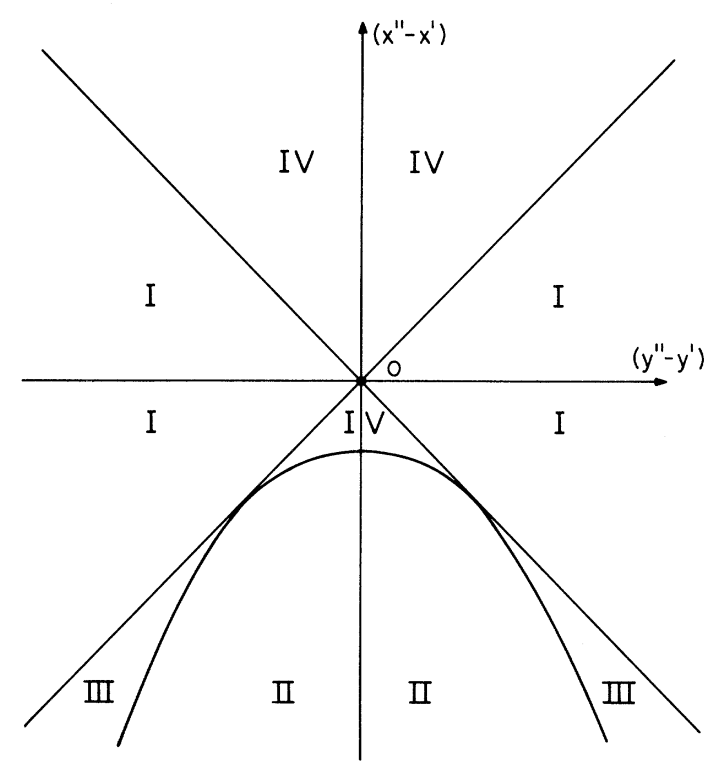

(a)

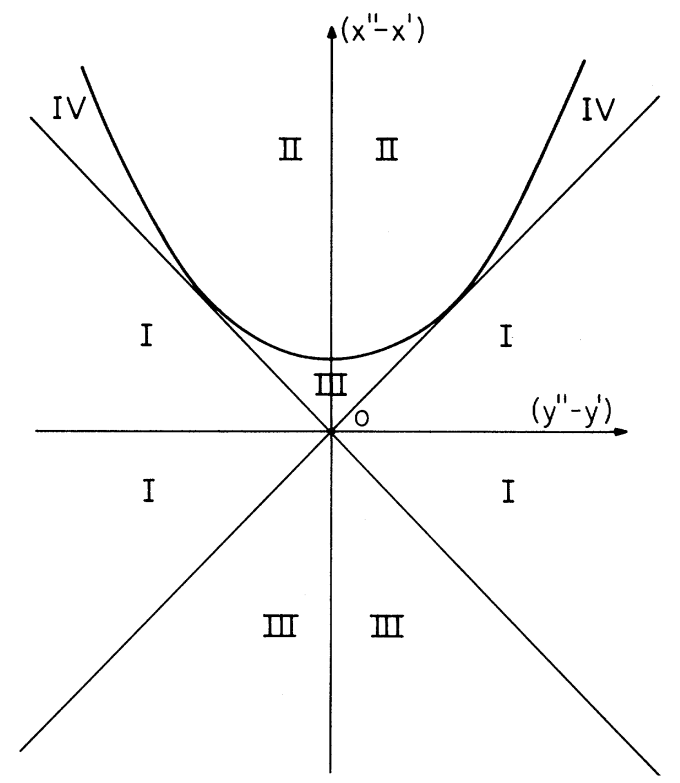

(b)

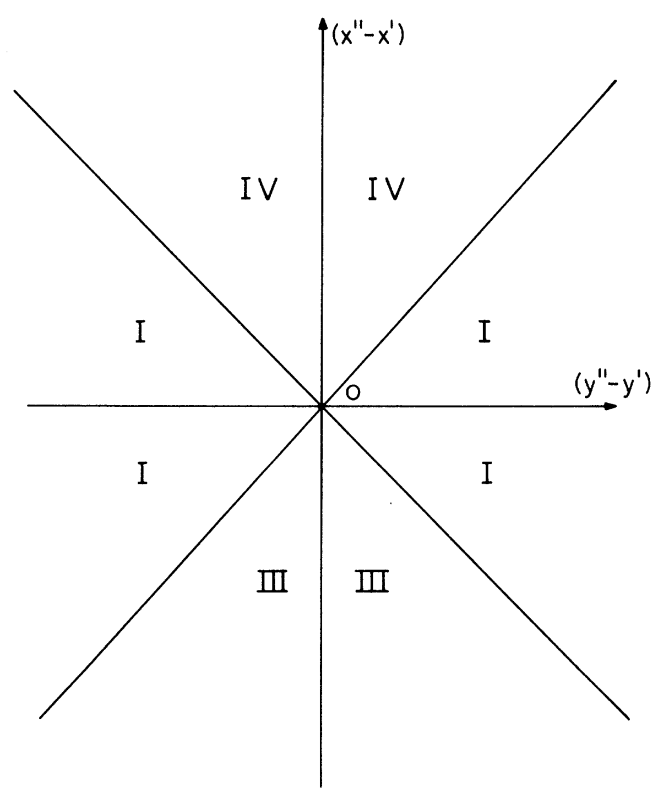

(c)

FIG. 2. (a) The figure illustrates the type of solutions arising when attempting to reach a final point in the $x y$ plane starting from the initial point $\left(x^{\prime}, y^{\prime}\right)$ at the center of the figure, in the case of $\alpha x^{\prime}>1$. There are two real and two imaginary solutions going to region I, four complex solutions to region II, four real solutions to region III, and four imaginary solutions to region IV. (b) The same as (a), but with $\alpha x^{\prime}<1$. (c) The same as (a), but with $\alpha x^{\prime}=1$. 
tions. The only exception to this is when $y^{\prime \prime}-y^{\prime}$, in which case $y(\tau)=0$ identically, and thus $\phi(\tau)=0$, and we are back to the de Sitter model of Ref. 6.

\section{CONTOUR ANALYSIS}

We now analyze the integral (4.10). We are interested in finding all the contours in the complex $N$ plane for which the integral (4.10) converges, and which leads to solutions of the Wheeler-DeWitt equation (2.11), or to Green's functions of the Wheeler-DeWitt operator (i.e., infinite or half-infinite contours). We will use the method of steepest descents to give an approximate evaluation of the integral along various contours. At the end of the section we shall reexpress the propagation amplitude (4.10) for some of the infinite contours as integral transforms of Airy functions, thus representing the propagation amplitude as superpositions of exact solutions to the Wheeler-DeWitt equation

\section{A. Contours for the exponential potential}

Consider first the pure exponential case, $\alpha=\beta$. The propagation amplitude (4.10) may be written

$$
G\left(x^{\prime \prime}, y^{\prime \prime} \mid x^{\prime}, y^{\prime}\right)=\int \frac{d N}{N} \exp \left[-\frac{R}{4} N-\frac{s^{2}}{8 N}\right) .
$$

The contour analysis for this integral is, in fact, identical to that for the propagation amplitude between fixed scale factors in the Kantowski-Sachs minisuperspace model studied in Ref. 8, so we will not repeat it here. The interested reader should consult Ref. 8 for further details. We will, however, quote from Ref. 8 the result of evaluating the integral along one particular contour, namely, the closed loop about the origin in the complex $N$ plane. In the case when the dominating saddle points (4.13) are Lorentzian, i.e., $s^{2}>0, R<0$ or $s^{2}<0, R>0$, one has, for the closed contour,

$$
\begin{aligned}
G\left(x^{\prime \prime}, y^{\prime \prime} \mid x^{\prime}, y^{\prime}\right) & =2 \pi i J_{0}\left[\left(-R s^{2} / 8\right)^{1 / 2}\right] \\
& \sim \cos \left[\left(-R s^{2} / 8\right)^{1 / 2}-\frac{\pi}{4}\right],
\end{aligned}
$$

where $J_{0}$ is the usual Bessel function, ${ }^{29}$ and the asymptotic form is valid for large $\left|R s^{2}\right|$.

\section{B. Contours for the cosh potential}

Consider next the case of the $\cosh (2 \phi)$ potential, $\beta=0$. The propagation amplitude (4.10) has the form

$G\left(x^{\prime \prime}, y^{\prime \prime} \mid x^{\prime}, y^{\prime}\right)=\int \frac{d N}{N} \exp \left(-\frac{\alpha^{2}}{24} N^{3}-\frac{R}{4} N-\frac{s^{2}}{8 N}\right)$.

Let us consider the possible infinite and half-infinite contours. Because the leading term in the exponent for large $|N|$ is $N^{3}$, there are three different directions in which the contour may go off to infinity. Moreover, an infinite con-

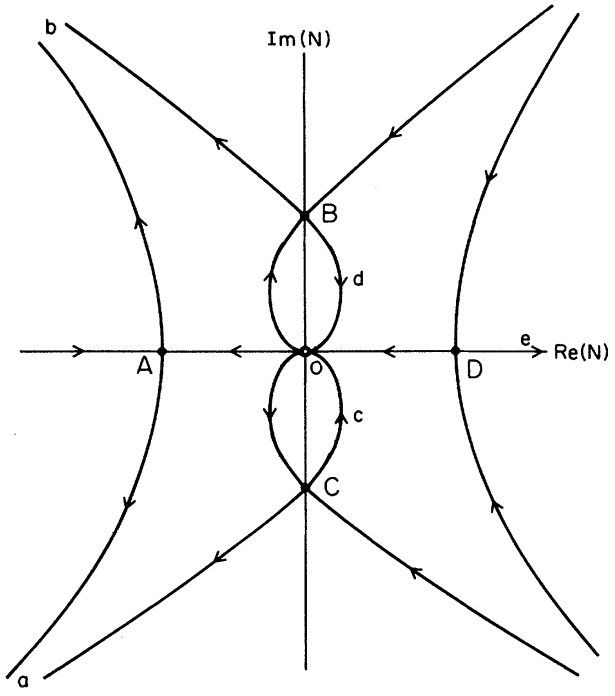

FIG. 3. The steepest-descent contours in the complex $N$ plane for the integral (5.3) in case Ia, i.e., for $s^{2}>0$ and either $R<0$ or $R>0$ and $3 R^{2}<\alpha^{2} s^{2}$. The saddle points $A$ and $D$ are real and $B$ and $C$ are imaginary. In this and the following figures, there is an essential singularity at the origin, and the arrows point downhill.

tour going between any two such regions may pass on either side of the essential singularity at the origin, with inequivalent results. The many possibilities for distinct infinite contours may, however, be reduced to combinations of just three infinite contours, which may be taken to be (i) the real Lorentzian contour passing to the left of the origin $N=i v-\epsilon$, where $v$ is real and $\epsilon>0$, (ii) the real Lorentzian contour passing to the right of the origin $N=i v+\epsilon$, and (iii) the "half-Lorentzian half-Euclidean" $\left(\frac{1}{2} L \frac{1}{2} E\right)$ contour coming down the positive imaginary

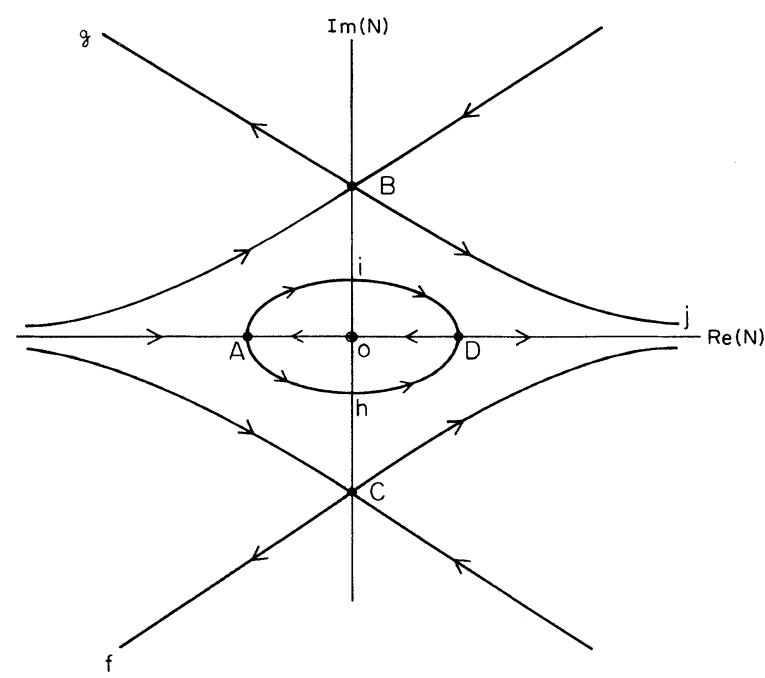

FIG. 4. The steepest-descent contours in case $\mathrm{Ib}$. The saddle points are the same, but $s^{2}>0, R>0$, and $3 R^{2}>\alpha^{2} s^{2}$. 
axis, going around the origin in the upper right quadrant and going out along the positive real axis. Similarly, for the half-infinite contours, all convergent possibilities may be obtained from the half-infinite contour running up the positive imaginary axis $N=i v^{2}$ combined with the infinite contours.

The integral (5.3) may be approximately evaluated along these contours, by first finding all the steepestdescent contours, and then distorting the above contours into sequences of steepest-descent contours. As in the analysis of the previous section, the analysis of the steepest-descent contours divides up into the four cases: I, II, III, and IV. However, case I divides further into two subcases, Ia and Ib, for the purposes of steepestdescent contours, although the saddle-point structure is the same. The five possible cases are as follows.

Ia: $R<0<s^{2}$, or $R>0$ and $3 R^{2}<\alpha^{2} s^{2}$. The steepestdescent contours are shown in Fig. 3. Contours (i)-(iii) distort into $a A b, a c O d b$, and $e O d b$, respectively. The half-infinite contour distorts into $O d b$.

Ib: $0<\alpha^{2} s^{2}<3 R^{2}, R>0$. The steepest-descent contours are shown in Fig. 4. Contours (i)-(iii) distort into fjhijg, $f j g$, and $j B g$, respectively. The half-infinite contour distorts into $O D j B g$.

II: $R^{2}+\alpha^{2} s^{2}<0$. The steepest-descent contours are shown in Fig. 5. Contours (i)-(iii) distort into $\mathrm{kOl}$, $k m p n l$, and $p n l$, respectively. The half-infinite contour distorts into $O A l$.

III: $s^{2}<0, R^{2}+\alpha^{2} s^{2}>0, R<0$. The steepest-descent contours are shown in Fig. 6. Contours (i)-(iii) distort into $q A r$, qstr, and $u t r$, respectively. The half-infinite contour distorts into $O B A r$.

IV: $s^{2}<0, R^{2}+\alpha^{2} s^{2}>0, R>0$. The steepest-descent contours are shown in Fig. 7. Contours (i)-(iii) distort into $v z x O y z w, v z w$, and $z B w$, respectively. The halfinfinite contour distorts into $\mathrm{Oy} A z B w$.

In each of the above cases it is easy to see from the figures which saddle point provides the dominant contribution. The action of each saddle point is readily found. Write the four saddle-point values of $N$, given by Eq. (4.14), as

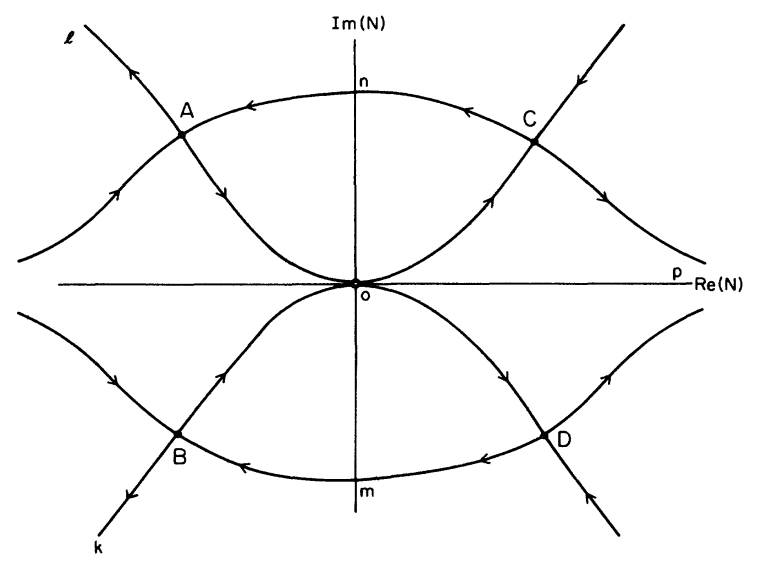

FIG. 5. The steepest-descent contours for case II, $R^{2}+\alpha^{2} s^{2}<0$. The four saddle points are all complex.

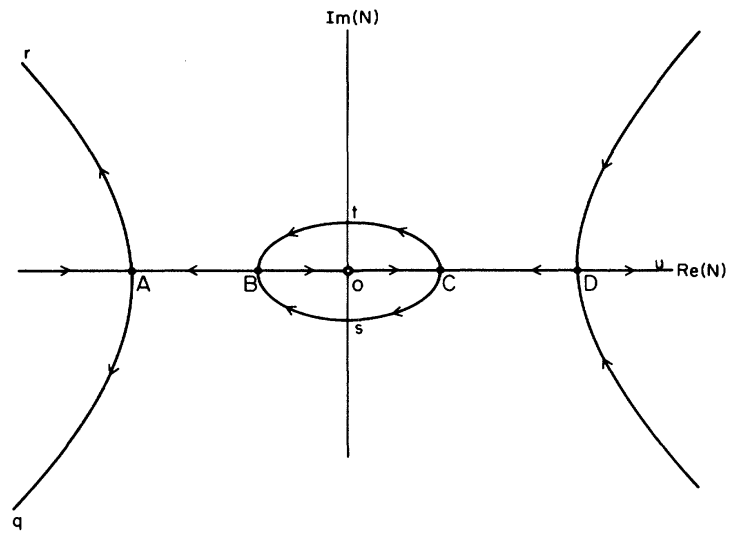

FIG. 6. The steepest-descent contours for case III, $s^{2}<0$, $R^{2}+\alpha^{2} s^{2}>0, R<0$. The four saddle points are all real.

$$
N=\frac{\epsilon_{1}}{\alpha}\left[-R+\epsilon_{2}\left(R^{2}+\alpha^{2} s^{2}\right)^{1 / 2}\right]^{1 / 2},
$$

where $\epsilon_{1}$ and $\epsilon_{2}$ take the values \pm 1 . If, in (5.4), we choose the cuts of the square roots to lie along the negative real axis and take the convention that $\sqrt{-1}=-i$, then in Figs. (3) $-(7)$, saddle point $A$ is $\left(\epsilon_{1}, \epsilon_{2}\right)=(-1,1), B$ is $(-1,-1), C$ is $(1,-1)$, and $D$ is $(1,1)$. The action for each saddle point is then

$$
\begin{aligned}
I= & \frac{\epsilon_{1}}{6 \alpha}\left[-R+\epsilon_{2}\left(R^{2}+\alpha^{2} s^{2}\right)^{1 / 2}\right]^{1 / 2} \\
& \times\left[2 R+\epsilon_{2}\left(R^{2}+\alpha^{2} s^{2}\right)^{1 / 2}\right] .
\end{aligned}
$$

The results of evaluating the integral along the above contours, to leading order in the saddle-point approximation, are shown in Tables $I(a)$ and $I(b)$.

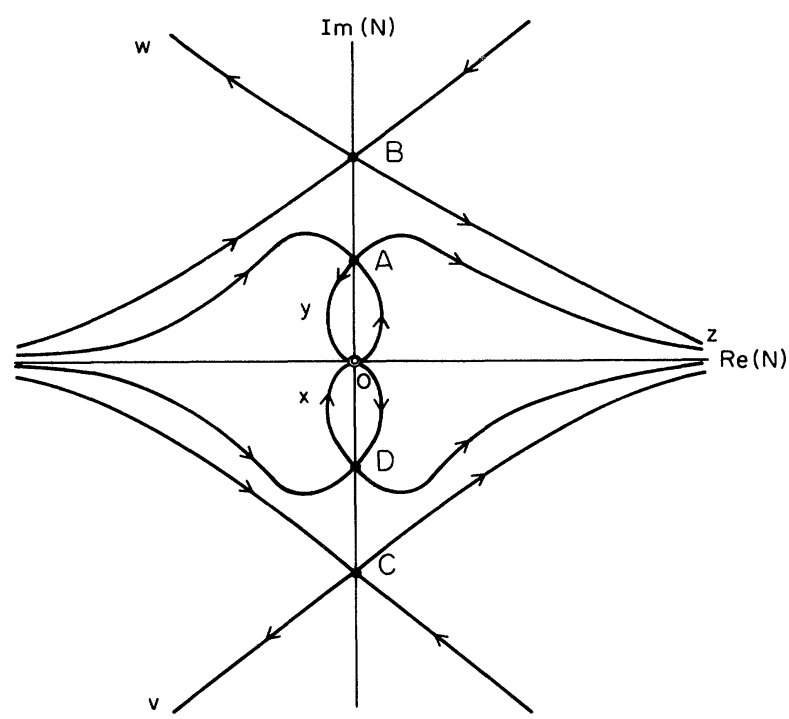

FIG. 7. The steepest-descent contours for case IV, $s^{2}<0$, $R^{2}+\alpha^{2} s^{2}>0, R>0$. The four saddle points are all imaginary. 
TABLE I. (a) This table shows the dominant contributions to the amplitude (5.3) along three infinite contours. Relative phases between different dominant contributions have been kept, but real prefactors have been dropped. The phase $\theta$ is given by $\theta=\pi / 2+1 / 2$ $\operatorname{arcos}\left[R /\left(\alpha^{2}\left|s^{2}\right|^{1 / 2}\right]\right.$, where arcos lies in the range $[0, \pi] . I_{A}$ denotes the action of saddle point $A$, and likewise for $B, C$, and $D$. The labeling of the saddle points is as shown in Figs. 3-7. (b) The bottom row shows the dominant contributions to the amplitude (5.3) for the half-infinite contour running up the positive imaginary axis.

\begin{tabular}{cccccc}
\hline \hline Contour & Case Ia & Case Ib & Case II & Case III & Case IV \\
\hline$N=i v+\epsilon$ & $e^{-I_{B}}+i e^{-I_{C}}$ & $e^{-I_{B}}+i e^{-I_{C}}$ & $e^{-I_{C}}-e^{i \theta^{-I_{D}}}$ & $e^{-I_{C}}$ & $e^{-I_{B}}+i e^{-I_{C}}$ \\
$N=i v-\epsilon$ & $e^{-I_{A}}$ & $e^{-I_{B}}+i e^{-I_{C}}-e^{i(\pi / 4)} e^{-I_{A}}$ & $e^{-I_{A}}+e^{-i \theta^{-I_{B}}}$ & $e^{-I_{A}}$ & $e^{-I_{A}}-i e^{-I_{D}+i e} e^{-I_{B}}-e^{-I_{C}}$ \\
$\frac{1}{2} L \frac{1}{2} E$ & $e^{-I_{B}}-e^{-i(\pi / 4)} e^{-I_{D}}$ & $e^{-I_{B}}$ & $e^{-I_{C}}$ & $e^{-I_{D}}$ & $e^{-I_{B}}$ \\
$N=i v^{2}$ & $e^{-I_{B}}$ & $e^{-I_{B}+e^{-i(\pi / 4)} e^{-I_{D}}}$ & $e^{-I_{A}}$ & $e^{-I_{B}}$ & $e^{-I_{A}}+i e^{-I_{B}}$ \\
\hline \hline
\end{tabular}

\section{Another integral representation}

The integral (5.3) may also be expressed as a superposition of the exact solutions (2.15) of the Wheeler-DeWitt equation. In those expressions the parameter $\mu$ is, in general, complex, and the behavior of the solutions (2.15) greatly depends on $\mu$. For instance, for $\mu<0$, the solutions $(2.15)$ are plane waves in the $y$ coordinate, whilst for $\mu>0$, they are unbounded. For the three infinite contours studied above, one can, in fact, rewrite the integral representation (5.3) of the propagation amplitude in another integral form which is more illuminating. Let

$$
\hat{X}=(1-\alpha x)(2 \alpha)^{-2 / 3}, \quad \hat{Y}=\alpha^{1 / 3} 2^{-2 / 3} y,
$$

and let $N \rightarrow \alpha^{2 / 3} 2^{-1 / 2} N$. Then the amplitude (5.3) takes the form

$$
\begin{aligned}
G=\int \frac{d N}{N} \exp [ & -\frac{N^{3}}{12}+\left(\hat{X}^{\prime \prime}+\hat{X}^{\prime}\right) \frac{N}{2} \\
& \left.+\frac{\left(\hat{X}^{\prime \prime}-\hat{X}^{\prime}\right)^{2}}{4 N}-\frac{\left(\hat{Y}^{\prime \prime}-\hat{Y}^{\prime}\right)^{2}}{4 N}\right] .
\end{aligned}
$$

Now introduce the identity

$$
\begin{aligned}
& \exp \left[-\frac{\left(\hat{Y}^{\prime \prime}-\hat{Y}^{\prime}\right)^{2}}{4 N}\right] \\
& \quad=\frac{N^{1 / 2}}{\pi^{1 / 2}} \int_{-\infty}^{+\infty} d w \exp \left[-i w\left(\hat{Y}^{\prime \prime}-\hat{Y}^{\prime}\right)\right] \exp \left(-N w^{2}\right)
\end{aligned}
$$

valid for all $N$ with a positive real part, and where the branch cut of the square root lies on the negative real axis. Along the contours (ii) and (iii) defined above [which may be deformed to lie entirely in the region $R e(N)>0$ ], Eq. (5.7) may be written

$$
\begin{gathered}
G=\pi^{-1 / 2} \int_{-\infty}^{+\infty} d w \exp \left[-i w\left(\hat{Y}^{\prime \prime}-\hat{Y}^{\prime}\right)\right] \\
\times G_{0}\left(\hat{X}^{\prime \prime}, \hat{X}^{\prime}, w^{2}\right)
\end{gathered}
$$

where

$$
\begin{aligned}
& G_{0}\left(\hat{X}^{\prime \prime}, \hat{X}^{\prime}, w^{2}\right) \\
& =\int \frac{d N}{N^{1 / 2}} \exp \left[-\frac{N^{3}}{12}+\left(\hat{X}^{\prime \prime}+\hat{X}^{\prime}-2 w^{2}\right) \frac{N}{2}\right. \\
& \left.+\frac{\left(\hat{X}^{\prime \prime}-\hat{X}^{\prime}\right)^{2}}{4 N}\right) .
\end{aligned}
$$

The significance of (5.9) is as follows: after some elementary rescalings of the variables, it is readily shown that $(5.10)$ is the propagation amplitude for the de Sitter minisuperspace model considered in Ref. 6, which can be evaluated exactly along contours (ii) and (iii) in terms of Airy functions. Along contour (ii), one thus obtains the result

$$
\begin{aligned}
G=\int_{-\infty}^{+\infty} & d w \exp \left[-i w \frac{y^{\prime \prime}-y^{\prime}}{2}\right] \\
& \times\left[\operatorname{Ai}\left(X^{\prime \prime}\right) \operatorname{Bi}\left(X^{\prime}\right)+\operatorname{Ai}\left(X^{\prime}\right) \operatorname{Bi}\left(X^{\prime \prime}\right)\right]
\end{aligned}
$$

(up to overall factors), where

$$
X=\left(1-\alpha x-w^{2}\right)(2 \alpha)^{-2 / 3} .
$$

Similarly, along contour (iii), one has

$$
\begin{aligned}
G=\int_{-\infty}^{+\infty} & d w \exp \left[-i w \frac{y^{\prime \prime}-y^{\prime}}{2}\right) \\
& \times\left[\operatorname{Ai}\left(X^{\prime \prime}\right)+i \operatorname{Bi}\left(X^{\prime \prime}\right)\right]\left[\operatorname{Ai}\left(X^{\prime}\right)+i \operatorname{Bi}\left(X^{\prime}\right)\right] .
\end{aligned}
$$

Equations (5.11) and (5.13) are clearly, as advertized, superpositions of the exact solutions (2.15), with the identification $\mu=-w^{2}$. Moreover, they are both Fourier transforms of the integral (5.10), which can also be interpreted as a propagation amplitude, as stated above.

For contour (i), which may be distorted so as to lie entirely in the region $\operatorname{Re}(N)<0$, one can repeat the calculation in a parallel fashion, using, instead of (5.8), the identity 


$$
\begin{aligned}
& \exp \left[-\frac{\left(\hat{Y}^{\prime \prime}-\hat{Y}^{\prime}\right)^{2}}{4 N}\right) \\
& =\frac{-i N^{1 / 2}}{\pi^{1 / 2}} \int_{-\infty}^{+\infty} d w \exp \left[-w\left(\hat{Y}^{\prime \prime}-\hat{Y}^{\prime}\right)\right] \exp \left(N w^{2}\right)
\end{aligned}
$$

valid for all $N$ with negative real part. In (5.14), the branch cut of the square root has been chosen to lie on the positive real axis. One thus obtains, for contour (i), the result

$G=\int_{-\infty}^{+\infty} d w \exp \left[-w \frac{y^{\prime \prime}-y^{\prime}}{2}\right] \operatorname{Ai}\left(X^{\prime \prime}\right) \operatorname{Ai}\left(X^{\prime}\right)$,

where $X$ is given here by

$$
X=\left(1-\alpha x+w^{2}\right)(2 \alpha)^{-2 / 3} .
$$

Again, (5.15) is a superposition of exact solutions (2.15), but with separation constant $\mu=w^{2}$. This superposition may be interpreted as a sum of Laplace transforms of an integral of the form (5.10). We have not been able to perform the analogous calculation for the case of the halfinfinite contour.

\section{THE NO-BOUNDARY WAVE FUNCTION}

So far we have been studying the propagation amplitude between fixed values of the minisuperspace coordinates $a$ and $\phi$ (or $x$ and $y$ ). Of greater interest from the point of view of practical quantum cosmology, however, are wave functions singled out by particular theories of initial conditions. In this section, therefore, we shall study the path-integral construction defining the "noboundary" wave function of Hartle and Hawking. ${ }^{15,16}$ As we shall soon see, this wave function is given, at least semiclassically, by a propagation amplitude between fixed minisuperspace coordinates with the initial coordinates set to certain values.

\section{A. No-boundary initial data}

In the no-boundary proposal of Hartle and Hawking, the wave function for the Universe is defined by a sumover-histories construction of the form

$\Psi_{\mathrm{NB}}\left[h_{i j}, \phi, \Sigma\right]=\sum_{M} \int \mathcal{D} g_{\mu \nu} \mathcal{D} \Phi \exp \left(-I\left[g_{\mu \nu}, \Phi\right]\right)$,

where $I$ is the Euclidean action of the gravitational field $g_{\mu \nu}$ and the matter fields $\Phi$, on a four-manifold $M$. The sum over manifolds is over compact manifolds $M$ which have as their only boundary the three-surface $\Sigma$ on which the arguments of the wave function are specified. The functional integral is over metrics $g_{\mu v}$ and matter fields $\Phi$ on $M$ which induce the values $h_{i j}$ and $\phi$ on $\Sigma .15,16$

A satisfactory definition of the sum over manifolds in (6.1) has never been given so, in practice, one normally considers each possible four-manifold separately, and this is what we shall do here. Because the gravitational part of the action in (6.1) is unbounded from below, the sum over metrics and matter fields is necessarily along a complex contour. ${ }^{3-10}$ No wholly satisfactory prescription uniquely fixing a contour for the no-boundary wave function has been given, to date, although a list of requirements that it should satisfy have been given in Ref. 5 . There is, therefore, a certain amount of ambiguity in the definition of the no-boundary wave function.

In addition to the above difficulties, a further issue arises in the actual implementation of the no-boundary proposal in minisuperspace models. Although the noboundary proposal is geometrically clear from the fourdimensional point of view, the $3+1$ decomposition entailed by minisuperspace demands that one impose certain "initial conditions" at the "bottom" of the fourgeometry. These initial conditions do not follow in a straight-forward way from the no-boundary proposal as stated above. Resting heavily on the semiclassical approximation to the path integral, a detailed discussion of an appropriate set of initial conditions was given in Ref. 8 (see also Ref. 32). The results of that discussion, as they affect the present paper, are summarized here. Appropriate initial conditions on the metric are partially obtained from purely geometrical considerations-insisting that the metric have no singularities at the bottom of the four-geometry. In more detail, the following requirements are useful.

(1) The boundary conditions must lead to a well-posed variational problem; i.e., the saddle-point condition $\delta I / \delta g_{\mu \nu}=0$ should yield in the minisuperspace field equations.

(2) The boundary conditions should ensure that the solutions to the field equations are regular everywhere on the given four-manifold and, in particular, they are regular at the bottom of the four-geometry.

(3) The conditions should be quantum-mechanically consistent (e.g., one should not have to fix a coordinate and its momentum simultaneously).

Further discussion of these points may be found in Ref. 8. These requirements are, it should be noted, very semiclassical in nature. Although this is perhaps not totally satisfactory, it is sufficient for present practical purposes.

Consider now the calculation of the no-boundary wave function for the scalar field model studied in the preceding sections. The first step is to fix the topology of the four-manifolds considered in the no-boundary amplitude. Recall that the three-surfaces $\Sigma$ are taken to be threespheres $S^{3}$. We therefore seek compact four-manifolds $M$ whose only boundary is a single three-sphere, $\partial M=S^{3}$. There are many such four-manifolds, but there is only one that will admit a metric Ansatz of the form (2.1), namely $B^{4}$, the closed ball in $\mathbb{R}^{4}$. For convenience, write the metric as

$$
d s^{2}=\sigma^{2}\left[d T^{2}+a^{2}(T) d \Omega_{3}^{2}\right]
$$

For this to be a regular metric on $B^{4}$, it is necessary to impose certain conditions at the center, namely, that the metric be that of flat space (in spherical coordinates) near $T=0$. This will be the case if we insist that $a^{2}(T)=T^{2}$ close to $T=0$, or, equivalently, 


$$
a(0)=0, \quad \frac{d a}{d T}(0)= \pm 1
$$

Now consider the scalar field. The Euclidean field equations and constraint may be written

$$
\begin{aligned}
& \frac{1}{a} \frac{d^{2} a}{d T^{2}}+2\left[\frac{d \phi}{d T}\right)^{2}+V(\phi)=0, \\
& \frac{d^{2} \phi}{d T^{2}}+\frac{3}{a} \frac{d a}{d T} \frac{d \phi}{d T}-\frac{1}{2} V^{\prime}(\phi)=0, \\
& \left(\frac{d a}{d T}\right)^{2}-1-a^{2}\left(\frac{d \phi}{d T}\right)^{2}+a^{2} V(\phi)=0 .
\end{aligned}
$$

If $a^{2}(T)=T^{2}$ for small $T$, then, of the two solutions to the scalar-field equation (6.5), one is regular and the other irregular. The irregular one may be eliminated by imposing the condition

$$
\frac{d \phi}{d T}(0)=0 \text {. }
$$

The conditions (6.3) and (6.7) ensure that requirement (2) holds.

Requirement (3) forbids both of the conditions (6.3) from being imposed simultaneously, since $d a / d T$ is proportional to the momentum conjugate to $a$. However, note that the constraint implies that the two conditions are, in fact, equivalent [given (6.7), and assuming that $V(\phi)$ remains bounded]. We may therefore choose to impose either one in the path integral, and the other will then hold at the saddle points. We choose to take the condition $a(0)=0$, and drop the derivative condition. This means that the geometries summed over in the full path integral will at least be closed at the bottom, but will generally not be regular (as one would expect).

Finally, consider requirement (1). This primarily concerns boundary terms in the action. For a manifold of topology $B^{4}$, the boundary term in the Einstein-Hilbert action (1.3) action contributes only at the final surface $T=1$. There is no "initial" surface, so no contribution from the boundary term at $T=0$. However, on inserting the metric Ansatz (6.2) and integrating the volume term by parts to get rid of the second-derivative terms, a boundary term does arise at $T=0$, namely, the term

$$
-\frac{1}{8 \pi G}\left[\int d^{3} x h^{1 / 2} K\right)_{T=0}=\left(-\frac{1}{2} a^{2} \frac{d a}{d T}\right]_{T=0} .
$$

The vanishing (or otherwise) of this term depends on the canonical data fixed at $T=0$. The candidate action we are now to take for the no-boundary amplitude is the action (2.3) plus the boundary term (6.8). Call it $\bar{I}$. To satisfy requirement (1), $\bar{I}$ must yield the correct field equations, when varied subject to the final conditions that the argument of the wave function is matched and the initial conditions (6.7) plus the condition $a(0)=0$. On performing the variation, it is readily shown that the field equations are obtained provided that the boundary term

$$
-\left[a^{3} \frac{d \phi}{d T} \delta \phi+\frac{1}{2} a^{2} \delta\left(\frac{d a}{d T}\right)\right]_{T=0}
$$

can be made to vanish. Equation (6.9) arises from (6.8), plus the terms obtained by integrating by parts in the variation of the action (2.3). Clearly this will indeed vanish with the condition $a(0)=0$, and requirement (1) is satisfied.

Next we need to express the conditions $a(0)=0$ and (6.7) in terms of the variables $x$ and $y$ used in the previous sections, defined by (2.4). From Eq. (2.4), one has

$$
\begin{aligned}
& a^{4}=x^{2}-y^{2}, \\
& \frac{d \phi}{d T}=\frac{1}{2 N} \frac{x \dot{y}-y \dot{x}}{\left(x^{2}-y^{2}\right)^{3 / 4}}, \\
& \frac{d a}{d T}=\frac{1}{2 N} \frac{x \dot{x}-y \dot{y}}{\left(x^{2}-y^{2}\right)^{1 / 2}},
\end{aligned}
$$

where the overdots on the right-hand side denote derivatives with respect to the time coordinate $\tau$ as defined in the metric (2.1). Fixing $d \phi / d T$ initially translates, via Eq. (6.11), into a rather complicated condition on $x$ and $y$, a condition it would be difficult to impose in the full path integral. However, recall that the condition (6.7) on $\phi$ was derived by purely classical considerations-from the requirement that the classical solutions be regular at the bottom of the four-geometry. It is therefore sufficient to find conditions on $x$ and $y$ that imply the condition (6.7) purely at the classical level. To this end, we shall argue that the initial conditions

$$
x(0)=0, y(0)=0
$$

do the job of imposing no-boundary initial data. Clearly (6.13) implies that $a(0)=0$, via (6.10). To see that (6.7) is satisfied, we need to use the classical field equations. The solution to the classical field equations satisfying the initial conditions (6.13) is given by (4.4) and (4.5), with $x^{\prime}=0, y^{\prime}=0$ (and with $N$ the solution to the appropriate algebraic equation, although this is not essential). Inserting this solution in Eq. (6.11), it is readily seen that, for small $\tau$, the denominator on the right-hand side goes like $\tau^{3 / 2}$, but the numerator goes like $\tau^{2}$. Equation (6.7) is therefore satisfied as $\tau$ goes to zero.

As an aside, we note what happens when one inserts the classical solutions for arbitrary $N$ into (6.12). One has

$$
\frac{d a}{d T}(0)= \pm \frac{1}{2 N}\left[\left(\alpha^{2}-\beta^{2}\right) N^{4}+2(R+2) N^{2}-s^{2}\right]^{1 / 2},
$$

where we have used the definitions of $R$ and $s^{2}$ in (4.11) with the initial conditions (6.13). At the saddle points, where the constraint is satisfied, one has

$$
\left(\alpha^{2}-\beta^{2}\right) N^{4}+2 R N^{2}-s^{2}=0
$$

from which it follows that

$$
\frac{d a}{d T}(0)= \pm 1
$$

As stated above, therefore, the geometries will only be regular at the saddle points.

We may now record the central result of this section: it is that the no-boundary amplitude is given by a path- 
integral expression of the form (4.1), with the initial conditions (6.13). Explicitly, one may write

$$
\begin{aligned}
& \Psi_{\mathrm{NB}}(a, \phi) \\
& \quad=G\left(x^{\prime \prime}=a^{2} \cosh (2 \phi), y^{\prime \prime}=a^{2} \sinh (2 \phi) \mid x^{\prime}=0, y^{\prime}=0\right) .
\end{aligned}
$$

As we saw in Sec. V, there are many contours which may be used to successfully define the amplitude (4.1), and thus, as noted at the beginning of this section, there will be many corresponding no-boundary wave functions, depending on how the contour is chosen. This conclusion was reached in Refs. 5-10. Here we will not give a complete or detailed discussion of all the possible contours, since such a discussion has already been given for very similar models in Refs. 5-10. Instead, we will discuss the no-boundary wave functions for some simple choices of contour. The tunneling wave function for this class of models is considered in Ref. 33 and we do not discuss it here.

\section{B. Predictions of classical spacetime}

Of primary concern are the regions in which the wave function predicts a classical spacetime. For this to be the case, a minimal requirement is that the wave function be predominantly of the form $e^{i S}$, where $S$ is a solution to the Lorentzian Hamilton-Jacobi equation

$$
(\nabla S)^{2}+U=0 \text {. }
$$

For then it may be argued $^{34}$ that the wave function is peaked about the set of classical solutions satisfying the first integral

$$
p=\nabla S \text {. }
$$

In the leading-order saddle-point approximation we are using here, the wave function (6.17) will be of the form

$$
\Psi_{\mathrm{NB}}(a, \phi) \sim e^{-I_{\mathrm{cl}}}
$$

(or possibly a sum of such terms) where $I_{\mathrm{cl}}$ is the Euclidean action of an, in general, complex solution, and satisfies the Euclidean Hamilton-Jacobi equation

$$
-\left(\nabla I_{\mathrm{cl}}\right)^{2}+U=0 \text {. }
$$

Write the complex action in terms of its real and imaginary parts: $I_{\mathrm{cl}}=I_{R}-i S$. One may thus see that the wave function (6.20) predicts a classical spacetime only in those regions for which

$$
\left|\nabla I_{R}\right| \ll|\nabla S| \text {. }
$$

For, if this condition holds, the wave function (6.19) is predominantly of the form $e^{i S}$, and (6.21) implies that $S$ will be an approximate solution to (6.18).

Next note that when $x^{\prime}=0=y^{\prime}$, the variables $R$ and $s^{2}$ (in terms of which the asymptotic forms of the wave functions are given), may be written

$$
\begin{aligned}
s^{2} & =-a^{4}, \\
R & =a^{2}[\alpha \cosh (2 \phi)+\beta \sinh (2 \phi)]-2,
\end{aligned}
$$

where, for convenience, we have dropped the double primes denoting final values.

\section{The no-boundary wave function for the exponential potential}

Now consider the no-boundary wave function for the exponential potential. Equations (5.2) and (6.23) yield the result

$$
\Psi_{\mathrm{NB}}(a, \phi) \sim e^{i S}+e^{-i S},
$$

where

$$
S \approx a^{2}\left(\frac{\alpha a^{2} e^{2 \phi}-2}{8}\right)^{1 / 2} .
$$

Equation (6.24) is valid in the region $a^{4}\left(\alpha a^{2} e^{2 \phi}-2\right) \gg 1$. Here, we have used the closed contour in the complex $N$ plane to define the no-boundary wave function. A different contour would lead to a different combination of the factors $e^{i S}, e^{-i S}$, but the consequent semiclassical predictions would be largely the same, since it can be argued that there is negligible interference between these two factors, and thus they may be treated separately. ${ }^{35}$ Equation (6.24) implies that the wave function is peaked about the set of classical solutions satisfying the first integrals

$$
-e^{\phi} a \frac{d a}{d t} \equiv p_{a}= \pm \frac{\partial S}{\partial a}, \quad e^{\phi} a^{3} \frac{d \phi}{d t} \equiv p_{\phi}= \pm \frac{\partial S}{\partial \phi} \text {. }
$$

Here, $t$ is the Lorentzian time coordinate defined in the metric (3.1). Upon integration, Eq. (6.26) defines a twoparameter subset of the three-parameter general solution to the Lorentzian field equations (3.3) and (3.4). One of these parameters is just $t_{0}$, the origin of unobservable parameter time, and is therefore irrelevant. The wave function (6.24) is therefore peaked about an effectively oneparameter family of solutions which do, in fact, correspond to a single curve in the $w z$ plane (in terms of the variables $w=\dot{a} / a, z=\dot{\phi}$ introduced in Sec. III). It is readily shown from $(6.26)$ that this curve is given by

$$
w=-z-\frac{\alpha}{4 z} \text {. }
$$

This curve is shown by the dashed line in Fig. 1. This family of solutions picked out by the no-boundary proposal has the key feature that it is amongst the set of solutions that end up on the attractor, at which there is power-law inflation.

\section{The no-boundary wave function for the cosh potential}

Now consider the $\cosh (2 \phi)$ potential. We seek the regions in minisuperspace in which the wave function is predominantly oscillatory, of the form $e^{i S}$. Consider the various regions, I, II, etc., discussed in the previous section. Because $x^{\prime}=0=y^{\prime}$, refer to Fig. 2(b) in the following discussion. It is convenient to introduce the parameter 


$$
\lambda \equiv \frac{\alpha^{2} s^{2}}{R^{2}}=-\frac{\alpha^{2} a^{4}}{\left[\alpha a^{2} \cosh (2 \phi)-2\right]^{2}},
$$

which governs the nature of the action (5.5) in each region. In terms of $\lambda$, Eq. (5.5) may be written

$$
\begin{aligned}
I= & \frac{\epsilon_{1}}{6 \alpha}|R|^{3 / 2}\left[-\frac{R}{|R|}+\epsilon_{2} \sqrt{1+\lambda}\right]^{1 / 2} \\
& \times\left[2 \frac{R}{|R|}+\epsilon_{2} \sqrt{1+\lambda}\right] .
\end{aligned}
$$

First of all, note that, with no-boundary initial data, $s^{2}=-a^{4}<0$, so we may immediately eliminate cases Ia and $\mathrm{Ib}$. All the other cases are consistent with $s^{2}<0$. Next, note that all the saddle points are real Euclidean in case III, so the wave function will be exponential in this region. Classical spacetime is therefore not predicted in region III.

We are therefore left with just the two cases, II and IV. Consider first region IV, in which $R>0,-1<\lambda<0$. All four saddle points are purely imaginary in this case, and the Euclidean action is therefore purely imaginary at every saddle point. Classical spacetime is, therefore, predicted for any choice of contour in region IV, provided the action is large enough for the steepest-descent approximation to be valid. This will be true if

$$
\alpha a^{2} \cosh (2 \phi) \gg 1 \text {. }
$$

Finally, consider region II, in which $-\infty<\lambda<1$. All four solutions are complex in this region. It is easily seen from Eq. (6.27) that the gradients of the real and imaginary parts of the action are comparable in magnitude for most values of $\lambda$, and classical spacetime is not, then a prediction. However, in the region $R>0$, with $\lambda$ very close to -1 , the action is almost entirely imaginary, and classical spacetime will be a prediction. The part of region II in which $\lambda$ is close to -1 to within some small amount $\delta$, say (i.e., $\lambda>-1-\delta$, where $\delta$ is small and positive), is the region outside the ellipse:

$$
\alpha^{2} y^{2}+\delta\left[\alpha x-2\left[1+\frac{1}{\delta}\right]\right]^{2}=4\left(1+\frac{1}{\delta}\right) .
$$

The focii of this ellipse lie on the $x$ axis in Fig. 2(b), and the ellipse touches the null lines at the same points the parabola touches. Although the action can have a rapidly varying imaginary part for all points outside this ellipse, it is only for those points which satisfy (6.29) that the steepest-descent approximation is really valid, and classical spacetime is predicted.

We may concisely summarize the behavior of the noboundary wave function for the $\cosh (2 \phi)$ potential as follows, referring to Fig. 2(b). We may think of Fig. 2(b) as the minisuperspace with coordinates $x$ and $y$, on which the no-boundary wave function is defined. The wave function exists for real $a$ and $\phi$ only in the future null cone of the origin, immediately excluding region I. Classical spacetime fails to be predicted in region III and in parts of region II. Classical spacetime is predicted only in the parts of regions II and IV satisfying condition (6.29).
In the classical region we may obtain asymptotic forms of the action and find the classical solutions picked out by the no-boundary proposal for certain choices of contour. Take, for example, the contour running down the imaginary axis to the right of the origin (in other examples this appears to define a reasonable no-boundary wave function). ${ }^{6,8}$ The form of the resulting wave function may be read off from Table $I(a)$. In the classical region, as described above, the action of the saddle points is almost entirely imaginary and may be calculated from (6.29). One thus obtains an oscillatory wave function of the form (6.24) (but with a slowly varying exponential prefactor) with

$$
S \approx \frac{\sqrt{\alpha}}{3} a^{3} \cosh ^{3} \phi .
$$

This corresponds to a set of classical trajectories which may be written

$$
w= \pm \sqrt{\alpha} \cosh ^{3} \phi e^{-\phi}, \quad z=-w \tanh \phi .
$$

This set of trajectories is qualitatively the same as the other solutions to the field equations, in that all of them go to the attractor, so there is obviously not anything special about the solutions picked out by the noboundary wave function.

\section{E. No-boundary saddle-point geometries}

To end this section, we discuss the nature of the saddle points in the path integral for the no-boundary amplitude. This ties in with our earlier discussions of the domain of $x$ and $y$ in Sec. II and IV. In terms of $x$ and $y$, the saddle-point configurations in the no-boundary amplitude are given by (4.4) and (4.5), with $x^{\prime}=0=y^{\prime}$, and with $N$ the solution to the appropriate algebraic equation. Explicitly,

$$
\begin{aligned}
& x(\tau)=-\alpha N^{2} \tau^{2}+\left(x^{\prime \prime}+\alpha N^{2}\right) \tau, \\
& y(\tau)=\beta N^{2} \tau^{2}+\left(y^{\prime \prime}-\beta N^{2}\right) \tau,
\end{aligned}
$$

where $0 \leq \tau \leq 1$. These are related to $a$ and $\phi$ by the inverse of the transformation (2.4), which is

$$
a^{2}=\left(x^{2}-y^{2}\right)^{1 / 2}, \quad \phi=\frac{1}{2} \operatorname{arctanh}\left[\frac{y}{x}\right] .
$$

Clearly, real $x$ and $y$ lead to real $a$ and $\phi$ only if $x>|y|$. Let us study the extent to which this is the case.

When $N$ is complex, $x$ and $y$ are complex, so $a$ and $\phi$ are complex and, as argued at the end of Sec. IV, the complex solutions have no obvious simple geometric interpretation in terms of real Lorentzian and real Euclidean geometries.

When $N$ is real, $x(\tau)$ and $y(\tau)$ are real. One has

$[x(\tau) \pm y(\tau)]=\left(x^{\prime \prime} \pm y^{\prime \prime}\right) \tau+(\alpha \mp \beta) N^{2} \tau(1-\tau)$.

We are only interested in final conditions in the region $x^{\prime \prime}>\left|y^{\prime \prime}\right|$. Also, $\alpha \geq|\beta|$. Since $N$ is real, it then follows from (6.37) that $x(\tau)>|y(\tau)|$ for all $\tau \in(0,1)$ and thus the solution in terms of $a$ and $\phi$ will indeed be real when $N$ is real. The corresponding geometries are smooth 
manifolds closing off a three-sphere, not unlike sections of four-sphere.

Now consider the case of $N$ purely imaginary. Because the Euclidean action is purely imaginary for these saddle points, one might have expected that the corresponding geometries and matter fields are real Lorentzian. This is not obviously the case, however. From (6.37) it is clear that there is a time $\tau_{0}$ given by

$$
\tau_{0}=1+\frac{x^{\prime \prime}-\eta y^{\prime \prime}}{(\alpha+\eta \beta) N^{2}},
$$

where $\eta=\operatorname{sgn}\left(\alpha y^{\prime \prime}+\beta x^{\prime \prime}\right)$, such that $x(\tau)>|y(\tau)|$ for $\tau>\tau_{0}$, and $x(\tau)<|y(\tau)|$ for $\tau<\tau_{0}$. Furthermore, using the saddle-point value of $N$, it is a little tedious but straightforward to show that $\tau_{0}$ lies in the interval $(0,1)$. The scale factor $a$ vanishes at $\tau=\tau_{0}$ and $\phi$ is infinite. For $\tau>\tau_{0}, a$ and $\phi$ are real so the geometry is real Lorentzian, but it shrinks to zero in a singular fashion at $\tau=\tau_{0}$. For $\tau<\tau_{0}, \phi$ and $a$ are complex, and the four-metric is complex. The geometry shrinks to zero in a regular way at $\tau=0$ [i.e., (6.3) is satisfied] but shrinks at zero in an irregular way at $\tau=\tau_{0}$. The (provisional) picture one therefore has is of a singular Lorentzian geometry matched at its singular beginning to a singularity on an otherwise regular closed four-geometry with a complex metric on it.

These saddle points may be simplified by complex diffeomorphisms, as described at the end of Sec. IV. In particular, the singularity at $\tau=\tau_{0}$ is readily avoided by taking $\tau$ around the point $\tau_{0}$ in the complex plane, making the solution completely regular [it is in this sense that the apparently singular nature of the solution is consistent with requirement (2) on the no-boundary data discussed in Sec. V A]. In the region $0<\tau<\tau_{0}$, we may write $a^{2}= \pm i|a|^{2}$, and $N^{2}=-|N|^{2}$. The complex fourmetric in this region may therefore be written

$$
d s^{2}= \pm i \sigma^{2}\left(|N|^{2} \frac{d \tau^{2}}{|a(\tau)|^{2}}+|a(\tau)|^{2} d \Omega_{3}^{2}\right] .
$$

It is therefore a real Euclidean four-metric with an overall imaginary conformal factor. Note that this is not inconsistent with the regularity condition at the bottom of the four-geometry:

$$
\pm 1=\frac{d a}{d T}=\frac{a}{N} \frac{d a}{d \tau}=\frac{1}{2 N} \frac{d}{d \tau}\left(a^{2}\right)
$$

This can be satisfied because both $N$ and $a^{2}$ are imaginary. In fact, close to the bottom of the four-geometry, Eq. (6.39) is just the flat metric (6.2) with an overall imaginary conformal factor, and this factor is readily absorbed by a complex diffeomorphism of $T$. It is in this sense that the saddle-point metric is that of flat Euclidean space at the bottom. With these simplifications obtained through complex diffeomorphisms, the final picture one has is as follows: the four-metric is that of completely regular flat, real Euclidean space near $\tau=0$ (with the scalar-field complex), it is real Lorentzian near $\tau=1$, and it is complex in the middle.

\section{SUMMARY AND CONCLUSIONS}

The purpose of this paper has been to perform a pathintegral analysis of a class of homogeneous isotropic minisuperspace models with a scalar field, in which the scalar-field potential is chosen so that the model is exactly soluble, or almost exactly soluble. The scalar-field potential was chosen to be a sum of exponentials, Eq. (1.9).

We began in Sec. II by writing down the action for scalar-field minisuperspace models. We showed that, in terms of the variables $x$ and $y$, the miniuperspace action with potential (1.9) is cast into a form in which the quantum-mechanical propagator (1.7) is exactly soluble. We wrote down the Wheeler-DeWitt equation for the model and found a set of exact solutions.

In Sec. III, we analyzed the classical Lorentzian solutions. In the case of a potential $\exp (2 \phi)$, the phase portrait possesses an attractor at which there is power-law inflation, and some proportion of (but not all) the solutions go to the attractor. For the $\cosh (2 \phi)$ potential, the exponential inflation is an attractor for all of the solutions.

We wrote down the path integral for the model in Sec. IV. Because the quantum-mechanical propagator (1.7) may be evaluated exactly, we found that the minisuperspace propagation amplitude (1.6) is given by a single ordinary integration over the lapse $N$. We proceeded to analyze this integral using the method of steepest descents. We began by finding all the saddle points of the integral, corresponding to solutions to the full Einstein-scalar-field equations. The solutions could be real Euclidean, real Lorentzian, or complex, depending on the boundary data, and we discussed the conditions under which each of these different types of solutions arose. These solutions are simple examples of the boundary-value problem for the Einstein field equations, about which very little is known in the general case. It is therefore very useful to have some simple examples, such as those found here, in which one can actually say something about the nature of the solutions for given boundary data. A better understanding of the Einstein boundary-value problem is likely to be important for future developments in quantum cosmology.

In Sec. V, we considered the evaluation of the propagation amplitude. We showed that the $\exp (2 \phi)$ case is essentially equivalent to the Kantowski-Sachs model studied in Ref. 8, and we used those results to evaluate the integral exactly for one particular contour. For the $\cosh (2 \phi)$ case, we found all the possible contours in the complex $N$ plane leading to solutions of the WheelerDeWitt equation or Green's functions of the WheelerDeWitt operator, and we evaluated the propagation amplitude along these contours, to leading order in the steepest-descent approximation. We also found an alternative integral representation of the propagation amplitude as a sum over the separation constant of the exact solutions to the Wheeler-DeWitt equation found in Sec. II.

In Sec. VI, we discussed the no-boundary proposal of Hartle and Hawking. We discussed the possible difficulties and ambiguities with this proposal in its application to particular models. We argued that, in our class 
of models, the no-boundary proposal could be implemented, at least semiclassically, by taking the initial conditions to be vanishing $x$ and $y$ in the propagation amplitude. We gave an approximate evaluation of the noboundary amplitude. We determined the regions in which the wave function is rapidly oscillatory, corresponding to classical spacetime, and we found the classical solutions picked out by this proposal. In the case of the $\exp (2 \phi)$ potential, the proposal picks out one of trajectories in the phase portrait which goes to the powerlaw inflationary attractor. It therefore picks out a favorable solution, in that only some proportion of the trajectories in the general solutions go to the attractor. For the $\cosh (2 \phi)$ potential, however, all of the trajectories in the general solution go to the attractor, so there is obviously not anything special about the solutions picked out by the no-boundary amplitude. Finally, we discussed the nature of the geometries corresponding to the saddle points of the no-boundary amplitude. The saddle points that appeared naively to be Lorentzian (because they had imaginary Euclidean action and imaginary $N$ ) turned out to be complex in terms of $a$ and $\phi$, due to the nature of the mapping from $x$ and $y$ to $a$ and $\phi$. Further investigation into some of the issues touched upon in this paper, such as the boundary-value problem and its importance for quantum cosmology, will be the subject of future publications.

\section{ACKNOWLEDGMENTS}

We are very grateful to Jorma Louko for many useful conversations. L.G. and G.M. would like to thank Massachusetts Institute of Technology (MIT), where most of this work was done, for warm hospitality during their visit. L.G. and G.M. were supported by the Spanish Ministerio de Educación y Ciencia. J.J.H. was supported in part by funds provided by the U.S. Department of Energy (DOE) under Contract No. DE-ACO2-76ER03069.
*Present address: Instituto de Óptica "Daza de Valdés," C.S.I.C., Serrano 121, 28006 Madrid, Spain.

${ }^{1}$ For some introductory/review material on quantum cosmology, see, for example, J. B. Hartle, in High Energy Physics 1985, proceedings of the Yale Summer School, New Haven, Connecticut, 1985, edited by M. J. Bowick and F. Gursey (World Scientific, Singapore, 1985); in Gravitation in Astrophysics (Cargese, 1986), proceedings of the NATO Advanced Study Institute, Cargese, France, 1986, edited by B. Carter and J. Hartle, NATO ASI Series B: Physics, Vol. 156 (Plenum, New York, 1987); J. J. Halliwell, in Proceedings of the Seventh Jerusalem Winter School for Theoretical Physics: Quantum Cosmology and Baby Universes, edited by S. Coleman, J. B. Hartle, and T. Piran (unpublished); in Proceedings of the Trieste Summer School on High Energy Physics and Cosmology, edited by Q. Shafi (unpublished); Institute for Theoretical Physics Report No. NSF-ITP-88-131 (1988) (unpublished); S. W. Hawking, in Relativity Groups and Topology $I I$, proceedings of the Les Houches Summer School, Les Houches, France, 1983, edited by B. DeWitt and R. Stora, Les Houches Summer School Proceedings, Vol. 40 (NorthHolland, Amsterdam, 1984). For an extensive bibliography, see J. J. Halliwell, Int. J. Mod. Phys. A 5, 2473 (1990).

${ }^{2} \mathrm{~J}$. B. Hartle, in Proceedings of the Seventh Jerusalem Winter School for Theoretical Physics: Quantum Cosmology and Baby Universes (Ref. 1).

${ }^{3}$ G. W. Gibbons, S. W. Hawking, and M. J. Perry, Nucl. Phys. B138, 141 (1978).

${ }^{4}$ J. B. Hartle and K. Schleich, in Quantum Field Theory and Quantum Statistics, Essays in Honor of the Sixtieth Birthday of E. S. Fradkin, edited by I. A. Batalin, G. A. Vilkovisky, and C. J. Isham (Hilger, Bristol, 1987); K. Schleich, Phys. Rev. D 36, 2342 (1987).

5 J. J. Halliwell and J. B. Hartle, Phys. Rev. D 41, 1815 (1990).

6J. J. Halliwell and J. Louko, Phys. Rev. D 39, 2206 (1989).

${ }^{7}$ J. J. Halliwell and J. Louko, Phys. Rev. D 40, 1868 (1989).

${ }^{8}$ J. J. Halliwell and J. Louko, Phys. Rev. D 42, 3997 (1990).

${ }^{9}$ J. B. Hartle, J. Math. Phys. 30, 452 (1989).

10J. J. Halliwell and R. Myers, Phys. Rev. D 40, 4011 (1989).

${ }^{11}$ J. J. Halliwell, Phys. Rev. D 38, 2468 (1988).

12J. J. Halliwell and J. B. Hartle, Phys. Rev. D 43, 1170 (1991).
${ }^{13}$ A. O. Barvinsky, Phys. Lett. B 175, 401 (1986); A. O. Barvinsky and V. N. Ponomariov, Phys. Lett. 167B, 289 (1986); C. Teitelboim, ibid. 96B, 77 (1980); Phys. Rev. D 25, 3159 (1982); 28, 297 (1983).

${ }^{14}$ J. B. Hartle, Phys. Rev. D 37, 2818 (1988); 38, 2985 (1988).

${ }^{15}$ S. W. Hawking, in Astrophysical Cosmology, edited by H. A. Brück, G. V. Coyne, and M. S. Longair (Pontifica Academia Scientarium, Vatican City, 1982); J. B. Hartle and S. W. Hawking, Phys. Rev. D 28, 2960 (1983).

${ }^{16}$ S. W. Hawking, Nucl. Phys. B239, 257 (1984).

${ }^{17}$ A. Linde, Zh. Eksp. Teor. Fiz. 87, 369 (1984) [Sov. Phys. JETP 60, 211 (1984)]; Nuovo Cimento 39, 401 (1984); Rep. Prog. Phys. 47, 925 (1984).

${ }^{18}$ A. Vilenkin, Phys. Rev. D 30, 509 (1984); 33, 3560 (1986); 37, 888 (1988).

${ }^{19}$ The literature abounds with minisuperspace models. Just a few of them are P. Amsterdamski, Phys. Rev. D 31, 3073 (1985); M. I. Beciu, Nuovo Cimento B 90, 223 (1985); R. Bergamini and G. Giampieri, Phys. Rev. D 40, 3960 (1989); S. del Campo and A. Vilenkin, Phys. Lett. B 224, 45 (1989); U. Carow-Watamura, T. Inami, and S. Watamura, Class. Quantum Grav. 4, 23 (1987); G. Esposito and G. Platania, ibid. 5, 937 (1988); L. Z. Fang and M. Li, Phys. Lett. 169B, 28 (1986); P. F. Gonzalez-Diaz, ibid. 159B, 19 (1985); J. J. Halliwell, Nucl. Phys. B286, 729 (1987); S. W. Hawking and J. C. Luttrell, Phys. Lett. 143B, 83 (1984); X. M. Hu and Z. C. Wu, ibid. 182, 305 (1986); S. R. Lonsdale and I. G. Moss, ibid. 189, 12 (1987); J. Louko and T. Vachaspati, ibid. 223, 21 (1989); Y. Okada and M. Yoshimura, Phys. Rev. D 33, 2164 (1986); S. Poletti, Class. Quantum Grav. 6, 1943 (1989); M. D. Pollock, Nucl. Phys. B324, 187 (1989); Y. G. Shen, Chin. Phys. L6, 43 (1989); A. Vilenkin, Phys. Rev. D 30, 509 (1984); Z. C. Wu, Phys. Lett. 146B, 307 (1984); Z. Zhuk, Class. Quantum Grav. 5, 1357 (1988). A guide to the literature including an extensive list of minisuperspace models may be found in J. J. Halliwell, in Proceedings of the Seventh Jerusalem Winter School for Theoretical Physics: Quantum Cosmology and Baby Universes (Ref. 1); in Proceedings of the Trieste Summer School on High Energy Physics and Cosmology (Ref. 1).

${ }^{20}$ C. W. Misner, in Relativity, edited by M. Carmeli, S. Fickler, 
and L. Witten (Plenum, New York, 1970).

${ }^{21}$ I. G. Moss, Ann. Inst. Henri Poincaŕe 49, 341 (1988).

${ }^{22}$ C. Kiefer, Zurich Report No. 90-0238, 1990 (unpublished).

${ }^{23}$ B. Berger, Phys. Rev. D 32, 2485 (1985); B. Berger and C. Vogeli, ibid. 32, 2477 (1985).

24J. J. Halliwell, Phys. Lett. B 185, 341 (1987).

${ }^{25}$ F. Lucchin and S. Matarrese, Phys. Rev. D 32, 1316 (1985).

${ }^{26}$ B. Ratra, Phys. Rev. D 40, 3939 (1989).

${ }^{27}$ R. A. Matzner and A. Mezzacappa, Found. Phys. 16, 227 (1986).

${ }^{28}$ Some papers dealing with the issues of quantum mechanics on restricted ranges are, for example, M. Carreau, E. Farhi and S. Gutmann, Phys. Rev. D 42, 1194 (1990); E. Farhi and S. Gutmann, Int. J. Mod. Phys. A 5, 3029 (1990); J. Klauder, in Relativity (Ref. 20); Phys. Rev. D 2, 272 (1970); N. Linden and M. Perry, Department of Applied Mathematics and Theoretical Physics report, 1990 (unpublished).

${ }^{29}$ Handbook of Mathematical Functions, Natl. Bur. Stand. Appl. Math. Ser. No. 55, edited by M. Abramowitz and I. A. Stegun (U.S. GPO, Washington, DC, 1965).
${ }^{30}$ K. V. Kuchař and M. P. Ryan, Phys. Rev. D 40, 3982 (1989).

${ }^{31}$ G. W. Gibbons and J. B. Hartle, Phys. Rev. D 42, 2458 (1990).

32J. Louko, Phys. Lett. B 202, 201 (1988); Ann. Phys. (N.Y.) 181, 318 (1988).

33J. Fort and A. Vilenkin (private communication).

${ }^{34}$ The peaking about classical trajectories is discussed in, for example, A. Anderson, Phys. Rev. D 42, 585 (1990); S. Habib, ibid. 42, 2566 (1990); J. J. Halliwell, ibid. 36, 3626 (1987); Phys. Lett. B 196, 444 (1987); T. P. Singh and T. Padmanabhan, Ann. Phys. (N.Y.) 196, 296 (1989); Class. Quantum Grav. 7, 411 (1990). A further requirement for classical behavior is decoherence of alternative histories. See, for example, M. Gell-Mann and J. B. Hartle, in Proceedings of the Third International Symposium on Foundations of Quantum Mechanics in the Light of New Technology, edited by S. Kobayashi (Japan Physical Society, Japan, 1990); C. Kiefer, Class. Quantum Grav. 4, 1369 (1987); M. Morikawa, Phys. Rev. D 40, 4023 (1989); T. Padmanabhan, ibid. 39, 2924 (1989). See also Ref. 35 below.

35J. J. Halliwell, Phys. Rev. D 39, 2912 (1989). 